\title{
Spectral theory of higher order differential operators by examples
}

\author{
Horst Behncke and Don B. Hinton
}

\begin{abstract}
Asymptotic integration has turned out to be a powerful method to determine the deficiency indices and spectra of higher order differential operators. Since the general method is by now well established we shall only outline this method and illustrate typical results and properties via examples. In addition to the calculation of deficiency indices, the location and multiplicity of the absolutely continuous spectrum will be found as well as showing the absence of singular continuous spectrum. Finite singular points will also be considered. For unbounded coefficients new results arise from competing terms of the operators.
\end{abstract}

Mathematics Subject Classification (2010). Primary 34A30, 34B20, 34L05; Secondary 34B05.

Keywords. Spectral theory, asymptotic solutions, absolutely continuous spectrum, singular spectrum, Titchmarsh-Weyl $m$-functions.

\section{Contents}

1 Introduction . . . . . . . . . . . . . . . . . . 361

2 The methods . . . . . . . . . . . . . . . . 362

3 Operators with bounded coefficients . . . . . . . . . . . 370

4 Operators with unbounded coefficients . . . . . . . . . . . . . 381

References . . . . . . . . . . . . . . . . . . . . . . 397

\section{Introduction}

In several papers the authors have investigated the spectral theory of higher order differential operators by means of asymptotic integration [2], [6], [3], [4], [8], [9], and [10]. These papers amply demonstrate the power of this method when applied to the analysis of the deficiency index, essential spectrum and absolutely continuous spectrum. Results from the far better known and simpler case of Sturm-Liouville 
operators suggest that most results are close to optimal with respect to their demands of smoothness and decay conditions.

This study is devoted to the spectral analysis of even order differential operators of the form

$$
L y=w^{-1}\left\{\sum_{k=0}^{n}(-1)^{k}\left(p_{k} y^{(k)}\right)^{(k)}-i \sum_{j=1}^{n}(-1)^{j}\left(\left(q_{j} y^{(j)}\right)^{(j-1)}+\left(q_{j} y^{(j-1)}\right)^{(j)}\right)\right\},
$$

and their odd order counterparts

$$
\begin{gathered}
L y=w^{-1}\left\{(-1)^{n} i\left(q_{n+1}\left(q_{n+1} y^{(n)}\right)^{\prime}\right)^{(n)}+p_{0} y+\sum_{k=1}^{n}(-1)^{k}\left(\left(p_{k} y^{(k)} y^{(k)}\right)^{(k)}\right.\right. \\
\left.\left.-i\left(q_{k} y^{(k)}\right)^{(k-1)}+\left(q_{k} y^{(k-1)}\right)^{(k)}\right)\right\} .
\end{gathered}
$$

These operators are supposed to act on the weighted $\mathscr{L}^{2}$-Hilbert spaces

$$
\mathscr{L}_{w}^{2}=\mathscr{L}^{2}((0, \infty), w),
$$

where the scalar product is defined with the weight function $w$. We will also consider the problem on $\mathscr{L}^{2}((-\infty, \infty), w)$ by means of the decomposition method developed in [8]. The coefficients are assumed to be real valued with a decomposition

$$
f=f_{0}+f_{1}+f_{2}+f_{3}, \quad f=p_{k}, q_{j},
$$

where $f_{0}$ is constant, $f_{1}$ twice differentiable, and $f_{2}$ once differentiable. In addition we require $f_{1}, f_{2}, f_{1}^{\prime}=o(1), f_{1}^{\prime \prime}, f_{2}^{\prime}, f_{3} \in \mathscr{L}^{1}$.

In our examples, however, we will hardly use the full generality of (1.3). For unbounded coefficients (1.3) will be modified to conditions on $\frac{f^{(k)}}{|f|}, k=1,2$.

To give a complete overview of the spectral properties of higher order is a hopeless task because of the multitude of cases [17] and [21]. Even the rather narrow class of fourth order operators exhibits a variety of spectral phenomena [2] and [6]. Thus we shall rather illustrate and interpret different spectral phenomena with the aid of examples along with comments on the general situation.

\section{The methods}

The analysis in this paper will always follow the procedure developed in [2], [6], [3], [4], [8], [9], and [10]. Thus we follow the steps: systems formulation, diagonalization and transformations, analysis of the dichotomy condition, asymptotic integration, The M-matrix, deficiency index, and spectra. 
2.1. Systems formulation. It is well known that higher order differential equations can be converted into first order systems. This transformation preserves linearity. For linear equations such as (1.1) or (1.2) it is most advantageous to employ quasiderivatives in order to minimize the demands on the differentiability of the coefficients. In the even order case, (1.1) they are defined by [24] and [25]:

$$
\begin{aligned}
y^{[k]} & =y^{(k)} \quad \text { for } 0 \leq k \leq n-1, \\
y^{[n]} & =p_{n} y^{(n)}-i q_{n} y^{(n-1)}, \\
y^{[n+1]} & =-\left(y^{(n)}\right)^{\prime}+i\left(q_{n} / p_{n}\right) y^{[n]}+\left(p_{n-1}-\left(q_{n}^{2} / p_{n}\right)\right) y^{(n-1)}-i q_{n-1} y^{(n-2)}, \\
y^{[n+k]} & =-y^{[n+k-1]^{\prime}}+p_{n-k} y^{[n-k]}+i\left(q_{n-k+1} y^{(n-k+1)}-q_{n-k} y^{n-k-1}\right), \\
& \quad 2 \leq k \leq n-1 .
\end{aligned}
$$

As one can see, the higher derivatives are made up from pieces of the operator. Then

$$
w L y=-\left(y^{[2 n-1]}\right)^{\prime}+i q_{1} y^{\prime}+p_{0} y .
$$

For odd order equations the quasiderivatives and systems formulation is given in [8]. So we restrict ourselves here to the even order case mainly. In order to write (1.1) in systems form let

$$
u=\left(y^{[0]}, \cdots, y^{[n-1]}, y^{[2 n-1]}, \cdots, y^{[n]}\right) .
$$

Then

$$
L y=z y
$$

becomes

$$
u^{\prime}=\left(\begin{array}{cc}
A & B \\
C & -A^{*}
\end{array}\right) u=\ell u .
$$

The non zero matrix elements of the $n$ by $n$ matrices are given by

$$
\begin{aligned}
A_{i, i+1} & =1, A_{n, n}=i \frac{q_{n}}{p_{n}}, \\
B_{n n} & =p_{n}^{-1}, \\
C_{11} & =p_{0}-z w, \\
C_{n, n} & =p_{n-1}-\frac{q_{n}^{2}}{p_{n}}, \\
C_{i, i} & =p_{i-1}, \quad i=2, \ldots, n-1, \\
C_{j, j+1} & =-C_{j+1, j}=i q_{j} .
\end{aligned}
$$


With the aid of the quasiderivatives the domain of the maximal operator $T^{*}$ is defined by

$$
D_{T^{*}}=\left\{y \in \mathscr{L}_{w}^{2} \mid y^{[k]} \text { is absolutely continuous for } 0 \leq k \leq 2 n-1, L y \in \mathscr{L}_{w}^{2}\right\} .
$$

The corresponding minimal operator $T$ is then $\left(T^{*}\right)^{*}$; see [25], [17], and [21]. The minimal operator $T$ is symmetric and its deficiency indices are defined by

$$
\operatorname{def} T=\left(\operatorname{dim} \mathcal{N}_{T^{*}-i}, \operatorname{dim} \mathcal{N}_{T^{*}+i}\right), \quad \mathcal{N}=\text { null space. }
$$

If the deficiency indices agree, $T$ will have selfadjoint extensions, which will always be denoted by $H$. One obtains these extensions by restricting $T^{*}$ by boundary conditions.

Hinton and Shaw [18] and Hinton and Schneider [19] have shown how to write the Hilbert space theory of $L$ or $T$ or systems like (2.2) directly in terms of such systems. In fact they study more general Hamiltonian systems:

$$
\mathfrak{J} u^{\prime}(x)=[z \mathcal{A}(x)+\mathscr{B}(x)] u(x), \quad \mathfrak{J}=\left(\begin{array}{cc}
0 & -1_{n} \\
1_{n} & 0
\end{array}\right) .
$$

In the case (2.2) one has

$$
\mathscr{B}=\left(\begin{array}{cc}
-C & A^{*} \\
A & B
\end{array}\right)
$$

and $\mathcal{A}_{11}=w$ and $\mathcal{A}_{i j}=0$ otherwise. In these papers [18] and [19] they show that the Hilbert space theory for (1.1), respectively $T, T^{*}$, is more or less identical with the Hilbert space theory defined for such systems. For odd order equations (1.2) the theory can be developed along the same lines, however, the Hamiltonian systems formulation requires particular care [18]. But finally one is led again to a system of the form

$$
u^{\prime}=e u \text {. }
$$

2.2. The characteristic polynomial. The simplest higher order differential operator is one with constant coefficients and $w=1$ on $\mathscr{L}^{2}(\mathbb{R})$. In this case the Fourier transform can be used to show that $T$ is unitarily equivalent to multiplication by

$$
\mathfrak{P}(\lambda)=\sum p_{k} \lambda^{2 k}+2 \sum q_{j} \lambda^{2 j-1}
$$

in the case of (1.1). For odd order operators (1.2) $T$ is unitarily equivalent to multiplication by

$$
\mathfrak{P}(\lambda)=q_{n+1}^{2} \lambda^{2 n+1}+\sum p_{k} \lambda^{2 k}+2 \sum q_{j} \lambda^{2 j+1} .
$$


We will therefore call $\mathfrak{P}$ the characteristic polynomial of (1.1), respectively (1.2). Thus the spectrum of $T$ is given by the range of $\mathfrak{P}$ on $\mathbb{R}$. In this case the spectrum $\sigma(T)$ of $T$ is absolutely continuous $\sigma_{\mathrm{ac}}$ and the discrete spectrum $\sigma_{\mathrm{d}}(T)$ is empty, i.e.

$$
\sigma(T)=\sigma_{\mathrm{ac}}(T)=\text { range of } \mathfrak{P}, \quad \sigma_{\mathrm{d}}(T)=\emptyset .
$$

A little thought shows that the absolutely continuous spectrum of multiplicity $k$, $\sigma_{\mathrm{ac}}(T, k)$, is given by

$$
\sigma_{\mathrm{ac}}(T, k)=\{z \in \mathbb{R} \mid \mathfrak{P}(\lambda)-z=0 \text { has exactly } k \text { real roots }\} .
$$

In (2.9) one has to disregard values $z$ for which $\mathfrak{P}(\lambda)-z=0$ and $\frac{d}{d \lambda} \mathfrak{P}=0$ for some $\lambda$. This defines the exceptional set $\mathcal{E}$, which is finite. From a functional analytic point of view $T$ can be considered as a function of the elementary self-adjoint operator of differentiation

$$
T_{0}=i \frac{d}{d x}, \quad T=\mathfrak{P}\left(T_{0}\right) .
$$

From a differential equation point of view one knows that the eigenfunctions of $T$ are "plane waves" of the form $\exp i \lambda x$ where $\lambda$ is a root of $\mathfrak{P}$, i.e.,

$$
T y=z y, \quad z \in \mathbb{R}, y=\sum a_{i} \exp i \lambda_{i} x .
$$

Now consider the same constant coefficient operator on $[0, \infty)$. For $(1.1)$ with all $q_{j}=0$ the sine- or cosine- transform can be used as above. However, if nontrivial $q_{j}$ are present, there is no easy way out. In fact for the even order operator $T$ one has def $T=(n, n)$, yet the Fourier transform does not lead anywhere. Even though there is a direct way out of this impasse, we shall not pursue this here, because this approach is bound to fail, when the coefficients are $x$ dependent. For this reason we shall pursue the analysis of the eigenfunctions of $L$, respectively $T^{*}$, because there is an intimate relationship between spectra and the associated eigenfunction. At least for Sturm-Liouville, operators one knows the following properties.

Bounded generalized eigenfunctions are associated to the absolutely continuous spectrum. Subordinate solutions with slow decay belong to the singular continuous or dense point spectrum. The eigenfunctions of a system (2.2) or more generally

$$
u^{\prime}=e_{u}
$$

will be determined by asymptotic integration. For this (2.12) will have to be transformed into Levinson form:

$$
v^{\prime}=(\Lambda(x)+R(x)) v, \quad \Lambda(x)=\operatorname{diag}\left(\lambda_{1}(x), \ldots, \lambda_{m}(x)\right), R(x) \in \mathscr{L}^{1} .
$$

First (2.12) has to be diagonalized by determining the eigenvalues and eigenvectors of $\mathcal{C}(2.12)$. Actually all this will only be based on the smooth part

$$
r_{s}=r_{0}+r_{1}+r_{2}
$$


of $\mathcal{C}$ (1.3). Here the part $\mathcal{C}_{i}$ is derived from the decomposition of the coefficients (1.3). While the eigenvalues are determined by the characteristic polynomial

$$
\operatorname{det}(\mathcal{C}(x, z)-\lambda),
$$

it is more advantageous to work with the characteristic Fourier polynomial

$$
\mathfrak{P}(x, z, \lambda)=\operatorname{det}(\mathcal{C}(x, z)+i \lambda I) .
$$

Expansion of the determinant yields with moderate effort in the even order case:

$$
\mathfrak{P}(x, z, \lambda)=\sum_{k=1}^{n} p_{k}(x) \lambda^{2 k}+\sum_{j=1}^{n} 2 q_{j}(x) \lambda^{2 j-1}+\left(p_{0}-w z\right),
$$

respectively,

$$
\mathfrak{P}(x, z, \lambda)=q_{n+1}^{2} \lambda^{2 n+1}+\sum_{k=1}^{n} p_{k}(x) \lambda^{2 k}+\sum_{j=1}^{n} 2 q_{j}(x) \lambda^{2 j-1}+\left(p_{0}-w z\right),
$$

in the odd order situation. In the remainder the discussion will be pursued only for even order operators, because the odd order situation will be almost identical. In addition define the limiting Fourier polynomial $\mathfrak{P}_{0}(z, \lambda)$ by $(2.15)$ with $p_{k}, q_{j}$ replaced by their limiting values.

2.3. Diagonalization. If $\lambda$ is an eigenvalue of $\mathcal{C}_{s}$, the corresponding eigenvector can be determined easily form the system of quasiderivatives. One just has to replace $y^{(k)}$ by $\lambda^{k}$ in the quasiderivatives. In addition the eigenvectors should be normalized by the factor

$$
M_{j}^{-\frac{1}{2}}=\partial_{\lambda} \mathfrak{P}\left(x, z, \lambda_{j}\right)^{-\frac{1}{2}}
$$

so that the eigenvector $\rho_{j}$ for the eigenvalue $\lambda_{j}$ is given by

$$
\rho_{j}(x, z)=M_{j}^{-\frac{1}{2}}\left(1, \lambda_{j}, \lambda_{j}^{2}, \ldots\right) .
$$

It is well known that the matrix $T$ formed with the eigenvectors of $\mathcal{C}_{s}$ as columns diagonalizes $\mathcal{C}_{s}$, the smooth part of $\mathcal{C}$,

$$
T^{-1} \mathcal{C}_{s} T=\operatorname{diag}\left(\lambda_{1}(x, z), \ldots, \lambda_{m}(x, s)\right)=\Lambda(x, z), \quad T=\left(\rho_{1}, \ldots, \rho_{m}\right),
$$

with $m=2 n$ or $2 n+1$.

Then the transformed system

$$
T v=u
$$

satisfies

$$
v^{\prime}=\left(\Lambda-T^{-1} T^{\prime}\right) v
$$


The term $-T^{-1} T^{\prime}$ arises, because $T$ depends on $x$. However, if $T$, respectively the coefficients of (1.1) or (1.2), are slowly varying, $T^{-1} T^{\prime}$ will be small. The assumptions (1.3) for example imply that the off-diagonal matrix elements of $\left(\Lambda-T^{-1} T^{\prime}\right)$ are square integrable. This is good, but generally not good enough. Thus a second diagonalization of the differentiable part of $\left(\Lambda-T^{-1} T^{\prime}\right)$ finally leads to the desired Levinson form (2.13). One should note that in this case the eigenvalues $\lambda_{i}(x, z)$ may be taken as the roots of the characteristic polynomial.

2.4. The dichotomy condition. Levinson's Theorem states that the solutions of the perturbed system (2.13) almost look like the solutions of the unperturbed system, $v^{\prime}=\Lambda v$, if the eigenvalues satisfy the dichotomy condition. In its simplest form it requires $\operatorname{Re}\left(\lambda_{i}(x, z)-\lambda_{j}(x, z)\right)$ to be of a constant sign for all $x \geq a$ and $i \neq j, i, j=1, \ldots, m$. This condition allows to estimate the solutions of the unperturbed system uniformly against each other. The actual dichotomy condition is a little weaker [14]. In our case this condition has to hold uniformly in the spectral parameter $z$ for $z \in \mathcal{K}$. Here $\mathcal{K}=\mathcal{K}_{\varepsilon}\left(z_{0}\right)=\left\{y \in \mathbb{C}|\operatorname{Im} z \geq 0| z-,z_{0} \mid<\varepsilon\right\}$ is a small set, which is to be analyzed with respect to its spectral properties for $T$, respectively $H$. To be more precise fix a spectral value $z_{0} \in \mathbb{R}$ so that the characteristic limiting Fourier polynomial $\mathfrak{P}_{0}$ has $2 n$, respectively $2 n+1$, roots. In the even order case the roots are $\alpha_{1} \pm i \beta, \ldots, \alpha_{r} \pm i \beta_{r}, \gamma_{2 r+1}, \ldots, \gamma_{2 n}$ with $\alpha, \beta, \gamma$ real. By continuity these extend to $2 n$ distinct roots of $\mathfrak{P}, \alpha_{1}(x, z) \pm$ $i \beta_{1}(x, z), \ldots, \alpha_{r}(x, z) \pm i \beta_{r}(x, z), \gamma_{2 r+1}(x, z), \ldots, \gamma_{2 n}(x, z)$. It is now easy to see that the $\alpha_{j}(x, z)+i \beta_{j}(x, z)$ lead to exponentially decaying and thus square integrable solutions. Thus the dichotomy condition has to be checked only for the essentially real eigenvalues $\gamma_{k}$. Since in first order

$$
\gamma_{j}\left(x, z_{0}+i \eta\right)=\gamma_{j}\left(x, z_{0}\right)+i\left(\partial_{\gamma} \mathfrak{P}\left(z_{0}, \gamma_{j}\right)\right)^{-1} w \eta,
$$

the dichotomy condition amounts to

$$
\partial_{\lambda} \mathfrak{P}_{0}\left(z_{0}, \gamma_{j}\right) \neq \partial_{\lambda} \mathfrak{P}_{0}\left(z_{0}, \gamma_{k}\right) \text { for } j \neq k, j, k=2 r+1, \ldots, 2 n \text {. }
$$

By invoking Bezout's theorem, it can be shown that (2.22) is violated at most in a countable set of spectral values $z$ with finitely many accumulation points. For spectral values $z_{0}$ outside this exceptional set $\mathscr{E}$ Levinson's theorem shows that the solutions of the initial equation (2.12) have the form

$$
u_{k}(x, z)=T(1+B)\left(e_{k}+r_{k}(x, z)\right) \exp \left(\int_{a}^{x} i \lambda_{k}(t) d t\right), \quad k=1, \ldots, 2 n,
$$

with $r_{k}(x, z) \rightarrow 0$ as $x \rightarrow \infty$ and $z \in \mathcal{K}_{\varepsilon}\left(z_{0}\right)$. Here $(1+B)$ stands for the second diagonalization and $e_{k}$ is the $k$-th unit vector. For the solutions of (1.1), respectively (1.2), this implies

$$
y_{k}(x, z)=M_{k}^{\frac{1}{2}}(x, z)\left(1+r_{k}(x, z)\right) \exp \left(i \int_{a}^{x} \lambda_{k}(t) d t\right) .
$$


If the eigenvalues $\lambda_{k}$ are of non real type $\lambda=\alpha_{j}+i \beta_{j}$, it can be shown that the $r_{k}$ are even analytic in $z$ for $z \in \mathcal{K}_{\varepsilon}$. This in turn will prevent dense point spectrum or the accumulation of eigenvalues.

2.5. The M-function. Once the eigenfunctions have been determined, a translation device is needed that relates properties of the eigenfunctions of a selfadjoint operator $H$ to that of its spectrum $\sigma(H)$. For Sturm-Liouville operators Weyl and Titchmarsh have developed the theory of the m-function as such a tool. This was later extended by Hinton and Shaw [18] and Hinton and Schneider [19] to Hamiltonian systems as a theory of the M-matrix. For even order half line problems (1.1) with associated operator $T$ with def $T=(n, n)$, the M-matrix is defined as follows. Fix a boundary condition $\alpha=\left(\alpha_{1}, \alpha_{2}\right)$ at the left hand end point 0 . The $n$ by $n$ boundary matrices satisfy

$$
\alpha_{1} \alpha_{1}^{*}+\alpha_{2} \alpha_{2}^{*}=1_{n} \text { and } \quad \alpha_{1} \alpha_{2}^{*}=\alpha_{2} \alpha_{1}^{*},
$$

and the selfadjoint extension $H_{\alpha}$ of $T$ is defined by the domain

$$
\left.D\left(H_{\alpha}\right)=\left\{u \in D\left(T^{*}\right) \mid\left(\alpha_{1}, \alpha_{2}\right) u(0)=0\right)\right\} .
$$

Let $Y_{\alpha}$ be the fundamental matrix of (2.2) with initial conditions

$$
Y_{\alpha}(0, z)=\left(\begin{array}{cc}
\alpha_{1}^{*} & -\alpha_{2}^{*} \\
\alpha_{2}^{*} & \alpha_{1}^{*}
\end{array}\right)
$$

Then there exists a unique matrix $M_{\alpha}(z), \operatorname{Im} z>0$, with

$$
Y_{\alpha}(x, z)\left(\begin{array}{c}
1 \\
M_{\alpha}(z)
\end{array}\right) \in \mathscr{L}_{w}^{2}(0, \infty) .
$$

It turns out that $M_{\alpha}(z)$ is analytic in the upper half plane and $\operatorname{Im} M(z) \geq 0$, i.e. $M_{\alpha}$ is a matrix valued Herglotz function. In the one dimensional case such functions are of the form

$$
m(z)=c_{1}+c_{2} z+\int_{\mathbb{R}}(\xi-z)^{-1} d \mu(\xi), \quad c_{1}, c_{2} \in \mathbb{R},
$$

for some measure $\mu$ and any Herglotz function arises in this way. Another form to generate such functions is through matrix elements of resolvents $(H-z)^{-1}$ of a selfadjoint operator $H$,

$$
m(z)=\left\langle(H-z)^{-1} x, x\right\rangle .
$$

If in this case $x$ is cyclic for $H$, the representation (2.28) for $m$ of (2.29) gives just the spectral measure of $H$. In the more general matrix case $\mu$ will be a matrix valued measure. The measure $\mu$ in (2.28) can be recovered from $M$ by

$$
\mu((a, b])=\pi^{-1} \lim _{\delta \rightarrow 0+} \lim _{\varepsilon \rightarrow 0+} \int_{\lambda_{1}+\delta}^{\lambda_{2}+\delta} \operatorname{Im}(M(\lambda+i \varepsilon)) d \lambda .
$$


The density of the absolutely continuous part is then

$$
d \mu_{\mathrm{ac}}(\lambda)=\pi^{-1} \lim _{\varepsilon \rightarrow 0+} \operatorname{Im} M(\lambda+i \varepsilon)=\pi^{-1} \operatorname{Im} M(\lambda+) .
$$

That the M-matrix from (2.29) is given almost by the matrix elements of the resolvent can be seen by computing the Greens-function (resolvent) of $H$. A more direct way is presented in [22]. There Remling also shows with $F_{\alpha}(x, z)=Y_{\alpha}(x, z)\left(\begin{array}{l}1 \\ M\end{array}\right)$,

$$
\left\langle F_{\alpha}(\cdot, z), F_{\alpha}(\cdot, z)\right\rangle=\frac{\operatorname{Im} M(z)}{\operatorname{Im} z}, \quad \operatorname{Im} z>0 .
$$

Here the $(i, j)$-matrix element is obtained from the scalar product of the $i$-th vector with the $j$-th vector. The systems scalar product in this case is of course defined via the matrix $\mathcal{A}$, which in our case is just $\mathcal{A}=\operatorname{diag}(w, 0, \ldots, 0)$. (2.31) can be rewritten as

$$
\operatorname{Im} M\left(z_{+}\right)=\lim _{\varepsilon \rightarrow 0+} \varepsilon\left\langle F_{\alpha}\left(\cdot, z_{+}+i \varepsilon\right), F_{\alpha}\left(\cdot, z_{+}+i \varepsilon\right)\right\rangle, \quad z=z_{+} \in \mathcal{K} \cap \mathbb{R}=I,
$$

and the existence of finite limits implies already that the spectrum is absolutely continuous in $I$, if there are not bound states. In this case $\frac{1}{\pi} \operatorname{Im} M\left(z_{+}\right)$would be the spectral density of $H$ in $I$. The $F_{\alpha}$, however, are not so easy to come by. If def $T=(n, n)$ and $\operatorname{Im} z>0$, let $V(x, z)$ be the $n$ by $2 n$ system of square integrable solutions of (2.2). Since there are exactly $n$ square integrable solutions, there exists an invertible $n$ by $n$ matrix $C(z)$ such that

$$
F_{\alpha}(x, z)=Y_{\alpha}(x, z)\left(\begin{array}{c}
1 \\
M_{\alpha}(x)
\end{array}\right)=V(x, z) C(z) .
$$

In this case (2.32) becomes

$$
\operatorname{Im} M\left(z_{+}\right)=\lim _{\varepsilon \rightarrow 0+} \varepsilon C^{*}(z)\langle V(., z) V(., z)\rangle C(z),
$$

and the matrix elements on the right hand side should be computed with the function from (2.24).

Now assume $F_{1}, \ldots, F_{k}$ are $z$ uniformly square integrable. Then the upper left hand $k$ by $k$ part in (2.32) vanishes. Since $\operatorname{Im} M$ is positive semi-definite only the lower $(n-k)$ by $(n-k)$ block of this matrix can differ from 0 . Thus the rank of $\operatorname{Im} M\left(z_{+}\right)$is $n-k$. This of course is just the multiplicity of the absolutely continuous spectrum. In asymptotic integration one has to restrict computations and estimates quite often to the interval $[a, \infty)$ with a large. Remling [22], however, has shown that such spectral results extend directly to $[0, \infty)$.

2.6. Spectral results. We can now summarize the key facts. For equation (1.1), respectively (1.2), determine the systems representation. Fix a set $\mathcal{K}_{\varepsilon}$ of spectral 
values $z$ for which the characteristic polynomial $\mathfrak{P}$ has only distinct eigenvalues and for which the dichotomy condition holds. The eigenfunctions for $z \in \mathcal{K}$ are then given by (2.23). Assume the roots of $\mathfrak{P}$ for $z \in \mathcal{K} \cap \mathbb{R}$ are $\alpha_{1}(x, z) \pm$ $i \beta(x, z), \ldots, \alpha_{r}(x, z) \pm i \beta_{r}(x, z), \gamma_{2 r+1}(x, z), \ldots, \gamma_{m}(x, z)$ with $\alpha, \beta, \gamma$ real and $m=2 n$ or $2 n+1$. Then the $\alpha_{j}+i \beta_{j}$ and $\gamma_{k}$-eigenfunctions are square integrable for $z+i \eta$ if $\frac{d \gamma}{d z}>0$. Thus with (1.3) we have the following two cases:

$$
\text { case (1.1): } \operatorname{def} T=(n, n) ; \quad \text { case (1.2): } \operatorname{def} T=(n+1, n) .
$$

As regards the spectrum we have that, if the square integrable eigenfunctions $u_{k}(x, z)$ depend continuously on $z$ for $y \in \mathcal{K}$, then $H$ has no absolutely continuous spectrum in $I=\mathcal{K} \cap \mathbb{R}_{0}$ for $z \in \mathcal{K}$. Thus the $z$-uniformly square integrable eigenfunctions contribute at most to the discrete spectrum. Only $(z+i \eta)$-eigenfunctions which lose their square integrability as $\eta \rightarrow 0$ will contribute to the absolutely continuous spectrum.

2.7. The decomposition method. Differential operators on $\mathbb{R}$ can be handled by introducing Dirichlet boundary conditions of order $2 n$, respectively $2 n+1$, at 0 . This allows us to consider the operator $T_{1}=T_{+} \oplus T_{-}$, where $T_{+}$and $T_{-}$are the restrictions of $T$. Now $T_{+}$and $T_{-}$can be treated as above. One should note that def $T_{1}=(2 n, 2 n)$ and that any two extensions of $T_{1}$, of which $H$ is one, have the same absolutely continuous spectrum. It is also possible to interpret $T_{1}$ as an operator of order $4 n$, respectively $2(2 n+1)$, on $\mathbb{R}_{+}$. This shows for the deficiency indices that

$$
\operatorname{def} T=\operatorname{def} T_{+}+\operatorname{def} T_{-}-(m, m), \quad m=2 n \text { or } 2 n+1 .
$$

This formula has been known for some time and goes back to Kodaira. As regards the absolutely continuous spectrum of $T$, the multiplicity of it is given by the number of square integrable $z$-eigensolutions, which lose their square integrability as $\operatorname{Im} z$ tends to 0 . So in a sense, the decomposition method applies to the spectrum as well.

\section{Operators with bounded coefficients}

The above results have the following consequences [3] and [8].

Theorem 3.1. Consider the operators $T(1.1)$ or $(1.2)$ on $[0, \infty)$ for which the coefficients satisfy the regularity assumptions (1.3). In the even order case the singular continuous spectrum $\sigma_{\mathrm{sc}}(H)$ of $H$ is empty and the absolutely continuous spectrum of $H$ agrees with that of the limiting constant coefficient operator. In particular $z \in \mathbb{R}$ belongs to the a.c. spectrum $H$ of multiplicity $k$ if the characteristic polynomial $\mathfrak{P}_{0}-z$ has $2 k$ real roots. Odd order operators on $\mathbb{R}$ are essentially selfadjoint. They have no singular continuous spectrum and their absolute continuous spectrum can be determined by the decomposition method, $T=T_{+} \oplus T_{-}$, and $\lambda \in \sigma_{\mathrm{ac}}(H, k)$ if $T_{+}$ 
and $T_{-}$have altogether $k$ independent $z+i \eta$ eigenfunctions which lose their square integrability as $\eta \rightarrow 0+$. Then for even order operators on $[0, \infty]$, def $T=(n, n)$ while for odd operators def $T=(n+1, n)$ or $(n, n+1)$ depending on the sign of $q_{n+1}$.

For operators on $\mathbb{R}$ use the decomposition method. Note that the coefficients on $\mathbb{R}_{+}$and $\mathbb{R}_{-}$need not have the same limits. By using the K.L.-transform [7] this result can be extended considerably [3].

Example 1 (The step potential on $\left.\mathscr{L}^{2}(\mathbb{R}): L y=(-1)^{n} y^{(2 n)}+a \chi_{[0, \infty)} y\right)$. The characteristic polynomial of $T_{+}$is $\lambda^{2 n}+a=z$. Only real roots of $\lambda$ of this polynomial contribute to the absolutely continuous spectrum for which also $\frac{\partial}{\partial \lambda} \mathfrak{P}\left(\lambda_{1}\right)>0$. Thus the a.c. part of $T_{+}$is $[a, \infty)$. For $T_{-}$the same argument applies. Thus $\sigma_{\mathrm{ac}}(H, 2)=$ $[a, \infty)$ and $\sigma_{\mathrm{ac}}(H, 1)=[0, a)$ if $a>0$. For $a<0$ one has $\sigma_{\mathrm{ac}}(H, 1)=[a, 0)$ and $\sigma_{\mathrm{ac}}(H, 2)=[0, \infty)$. For odd order operators $L y=(-1)^{n} i y^{(2 n+1)}+a \chi_{[0, \infty)} y$ a similar analysis shows $\sigma_{\mathrm{ac}}(H, 1)=\mathbb{R}$.

Example 2 (The bump potential). In quantum mechanics the bump potential is studied in connection with the tunnel effect. There it is shown that a plane wave solution will have a reflected and transmitted part. In the more general case of

$$
L y=(-1)^{n} y^{(2 n)}+b \chi_{[-1,1]} y, \quad b>0, \text { on } \mathscr{L}^{2}(\mathbb{R}),
$$

this is not different. A detailed form can be computed for $n=2$ for example. In this case $H \geq 0$ so $\sigma(H) \subseteq[0, \infty)$. The decomposition method shows $\sigma_{\mathrm{ac}}(H, 2)$ $=[0, \infty)$ and $\sigma_{\mathrm{sc}}(H)=\emptyset$. We now show $H$ has no eigenvalues. Suppose $\lambda$ is an eigenvalue of $H$ with eigenfunction $\psi$. For $x<-1$ or $x>1$ the function $\psi$ will decay exponentially at $\pm \infty$ since it is in $\mathscr{L}^{2}(\mathbb{R})$ and satisfies a differential equation with constant coefficients. In the following calculations the exponential decay justifies the integrations by parts. First of all we have

$$
\langle H \psi, \psi\rangle=\int_{-\infty}^{\infty}\left[\left(\psi^{(n)}\right)^{2}+b \chi_{[-1,1]} \psi^{2}\right] d t=\lambda \int_{-\infty}^{\infty} \psi^{2} d t .
$$

Secondly, we have by multiplication of $(-1)^{n} \psi^{(2 n)}+b \chi_{[-1,1]} \psi=\lambda \psi$ by $t \psi^{\prime}(t)$ that

$$
\int_{-\infty}^{\infty}\left[\left(\psi^{(n)}\right)\left(t \psi^{\prime}\right)^{(n)}+b \chi_{[-1,1]} t \psi \psi^{\prime}\right] d t=\lambda \int_{-\infty}^{\infty} t \psi \psi^{\prime} d t .
$$

Integration by parts yields from (3.3) that

$$
\int_{-\infty}^{\infty}\left[\left(n-\frac{1}{2}\right)\left(\psi^{(n)}\right)^{2}+b \chi_{[-1,1]} t \psi \psi^{\prime}\right] d t=\frac{-\lambda}{2} \int_{-\infty}^{\infty} \psi^{2} d t .
$$

This shows that

$$
\int_{-\infty}^{\infty} b \chi_{[-1,1]} t \psi \psi^{\prime} d t=\frac{b}{2}\left[\psi(1)^{2}+\psi(-1)^{2}-\int_{-1}^{1} \psi^{2} d t\right] .
$$


Adding (3.2) to twice (3.4) gives

$$
(2 n) \int_{-\infty}^{\infty}\left(\psi^{(n)}\right)^{2} d t+b\left[\psi(1)^{2}+\psi(-1)^{2}\right]=0 .
$$

From this we conclude that $\psi$ is the zero function. This contradiction shows $H$ has no eigenvalues. The derivation above can be generalized and leads to the virial theorem, which will be discussed in a future publication by the authors.

This result will most likely be true also for more general compactly supported bumps.

Example 3. Consider the compact support potential

$$
L y=(-1)^{n} y^{(2 n)}+q(t) y \quad \text { on } \mathscr{L}^{2}(\mathbb{R}),
$$

where $q \in \mathscr{L}(\mathbb{R})$ and has compact support with $\int_{-\infty}^{\infty} q(t) d t<0$. The decomposition method shows $\sigma_{\mathrm{ac}}(H, 2)=[0, \infty)$ and $\sigma_{\mathrm{sc}}(H)=\emptyset$. We now show that $H$ must have at least one negative eigenvalue under these conditions. Suppose $H$ has no negative eigenvalues. Then we have $\sigma(H)=[0, \infty)$, and $\langle H \psi, \psi\rangle \geq 0$ for all $\psi$ in the domain of $H$. Let now $\varphi$ be a $C^{\infty}$ function on $\mathbb{R}$ with support $[-2,2]$ and $\varphi(t)=1$ on $[-1,1]$. Define, for $b>0, \psi(t)=\varphi(b t)$. Then $\psi$ has support $[-2 / b, 2 / b]$ and $\psi$ is in the domain of $H$. Thus

$$
\begin{aligned}
\langle H \psi, \psi\rangle & =\int_{-\infty}^{\infty}\left[\left(\psi^{(n)}(t)\right)^{2}+q(t) \psi^{2}(t)\right] d t \\
& =\int_{-2}^{2} b^{2 n-1}\left(\varphi^{(n)}(x)\right)^{2} d x+\int_{-2 / b}^{2 / b} q(t) \varphi^{2}(b t) d t .
\end{aligned}
$$

Now $\int_{-2 / b}^{2 / b} q(t) \varphi^{2}(b t) d t \rightarrow \int_{-\infty}^{\infty} q(t) d t<0$ as $b \rightarrow 0$. Hence $\langle H \psi, \psi\rangle$ is negative for $b$ sufficiently small which is contrary to $\langle H \psi, \psi\rangle \geq 0$ for all $\psi$ in the domain of $H$. This contradiction shows $H$ has a negative eigenvalue.

In quantum mechanics a student will generally study the square well potential, because it serves as a model for deuterium. The higher order cases do not have such a nice application, but the next example shows that a very negative well may have a large number of bound states.

Example 4. Consider the $2 n$-th order square well

$$
L y=(-1)^{n} y^{(2 n)}-a^{2 n} \chi_{[-1,1]} y, \quad a>0, \text { on } \mathscr{L}^{2}(\mathbb{R}) .
$$

Then $\sigma_{\mathrm{ac}}(H, 2)=[0, \infty), \sigma_{\mathrm{sc}}(H)=\emptyset$. Example 3 shows that $H$ has at least one negative eigenvalue. For $n=2$ it can be shown by a direct calculation that there are 
no embedded bound states in the continuum spectrum. For $n>2$ we conjecture that there are also no embedded bound states in the continuum spectrum.

In order to analyze the general case, we could use perturbation methods. Write

$$
A=(-1)^{n}\left(\frac{d}{d x}\right)^{2 n}
$$

and

$$
B=\chi[-1,1] .
$$

Then $L y=\lambda y$ for $\lambda<0$ can be rewritten as

$$
\left(1-a^{2 n}(A-\lambda)^{-\frac{1}{2}} B(A-\lambda)^{-\frac{1}{2}}\right) y=0
$$

or $a^{-2 n} \in \sigma\left(B(A-\lambda)^{-1} B\right)$. Since $B(A-\lambda)^{-1} B$ is non finite rank trace class, it will have an arbitrary large number of bound states which accumulate at 0 . More definitive statements can be made however.

For $n=2$ one can show by direct, albeit cumbersome, computation, the eigenvalues of odd bound states of $L y=\lambda y$, which lie in $-a^{4}<\lambda<0$, satisfy the transcendental equation,

$$
e^{-2 \beta} \frac{\beta^{2}-\sqrt{2} \alpha \beta+\alpha^{2}}{\beta^{2}+\sqrt{2} \alpha \beta+\alpha^{2}}=-\frac{A \tan \beta-B}{B \tan \beta+A},
$$

where $\alpha=|\lambda|^{1 / 4}, \beta=\left|\lambda+a^{4}\right|^{1 / 4}, A=\beta^{2}-\sqrt{2} \alpha \beta+\alpha^{2}, B=\beta^{2}+\sqrt{2} \alpha \beta+\alpha^{2}$. Further analysis shows the number $N\left(a^{4}\right)$ of eigenvalues in the interval $-a^{4}<$ $\lambda<0$, satisfies $N\left(a^{4}\right) / a \rightarrow 1 / \pi$ as $a \rightarrow \infty$. A similar formula holds for the even eigenfunctions. This equation gives a method to approximate the eigenvalues accurately, but becomes unwieldy for higher order equations.

However, an asymptotic formula for the number of eigenvalues can be obtained by operator methods. As noted above, $\sigma_{\mathrm{ac}}(H, 2)=[0, \infty), \sigma_{\mathrm{sc}}(H)=\varnothing$. So it remains to determine the negative eigenvalues. Since $H$ is an even and real operator the eigenfunctions will be either even or odd and may be chosen real valued. We will consider the restriction of $H$ to the even (odd) eigenfunctions where the results for the odd eigenfunctions will be given in brackets. Let $L_{00}$ be the operator $L$ with Dirichlet boundary conditions at -1 and 1, i.e. $y^{(k)}( \pm 1)=0,0 \leq k \leq 2 n-1$. $L_{00}$ has deficiency index $4 n$. It has a selfadjoint even (odd) extension $L_{1}$ defined by the even (odd) boundary conditions $y^{(2 k+1)}( \pm 1)=0,0 \leq k \leq n-1\left(y^{(2 k)}( \pm 1)=\right.$ $0,0 \leq k \leq n-1)$ so that $L_{1}$ decomposes into a direct sum $L_{1}=L_{11}+L_{12}+L_{13}$ of the corresponding operators on $(-\infty,-1],[-1,1]$, respectively $[1, \infty)$. This shows in particular from Behncke [1] for the number of bound states $N\left(a^{2 n}\right)$ of $H$ respectively, $N_{1}\left(a^{2 n}\right)$ of $L_{1}$, that

$$
\left|N\left(a^{2 n}\right)-N_{1}\left(a^{2 n}\right)\right| \leq 2 n
$$

because the resolvents are perturbations of rank $2 n$ of each other. Now $L_{13}=L_{03}^{n}$ where $L_{03}=-d^{2} / d x^{2}$ with the boundary condition $y^{\prime}(1)=0$. Clearly $L_{03}$ and 
thus $L_{13}$ has no bound states. This holds likewise for $L_{11}$. Obviously we have then

$$
\begin{aligned}
N_{1}\left(a^{2 n}\right) & =\text { number of bound states of } L_{12}, \\
& =\text { number of bound states of } L_{n 0} \leq a^{2 n}, \\
& =\text { number of bound states of } L_{10} \leq a^{2},
\end{aligned}
$$

where $L_{n 0}=\left(-d^{2} / d x^{2}\right)^{n}$ with even (odd) boundary conditions. One can easily determine the eigenfunctions of $L_{10}$ even (odd). They are $\cos \left(n+\frac{1}{2}\right) \pi x \quad(\sin n \pi x)$ and thus, adding both even and odd eigenvalues,

$$
N_{1}\left(a^{2 n}\right)=\left\lfloor\frac{a}{\pi}-\frac{1}{2}\right\rfloor+\left\lfloor\frac{a}{\pi}\right\rfloor
$$

where $\lfloor\cdot\rfloor$ is the greatest integer function.

Remark 3.2. The method of proof can be extended to more general deep wells. It can be shown by direct calculations that the third order square-well has no bound states. This will hold most likely also for the $(2 n+1)$-st order square well.

In the theory developed above we had $w=1$ so that in the case of bounded coefficients the spectral term $z w$ could be used to enforce the dichotomy condition outside the exceptional set $\mathcal{E}$. If this is not valid any more the dichotomy condition has to be assumed, but it can be enforced by a variation of $p_{0}$.

Example 5. We consider now the situation $p_{n}=1=\left|p_{0}\right|, w=o(1)$ where all coefficients are constant modulo integrable terms.

$$
p_{k}(x)-c_{k}, \quad q_{j}(x)-d_{j} \in \mathscr{L}^{1} \quad k=0, \cdots, n-1, j=1, \cdots, n .
$$

Moreover assume that the limiting Fourier polynomial

$$
\mathfrak{P}(\lambda)=\sum \lambda^{2 k} c_{k}+2 \sum d_{j} \lambda^{2 j-1}
$$

has $2 n$ distinct roots. Let $\mu_{1}, \ldots, \mu_{2 k}$ denote the real roots and $\alpha_{r} \pm i \beta_{r}, r=$ $1, \ldots, n-k$ the non real roots. Assume $w>0$ is non-integrable. The dichotomy condition now requires $\operatorname{Re} \partial_{\lambda} \mathfrak{P}(\mu) \neq \operatorname{Re} \partial_{\lambda} \mathfrak{P}(\tilde{\mu})$ if $\mu$ and $\tilde{\mu}$ are roots of $\mathfrak{P}$ with $\operatorname{Re} \mu=\operatorname{Re} \tilde{\mu}$. Then $\operatorname{def} T=(n, n)$ and $\sigma(H, k)=\mathbb{R}$, because $k$ square integrable functions lose their square integrability as $\operatorname{Im} z \rightarrow 0+$. This is shown as in [10]. If $w$ is integrable def $T=(n+k, n+k)$ and $\sigma(H)$ is discrete, because all square integrable eigenfunctions are $z$-uniformly square integrable.

Power coefficients have been quite popular with mathematicians with differential operators. For this reason we will call a function $f$ to be of approximate power type if

$$
f=f_{0} x^{\alpha_{f}}\left(1+h_{f}\right), \quad h_{f} \in \mathcal{F}_{l},
$$

where

$$
\widetilde{F}_{l}=\left\{f \mid f^{(k)}=o\left(x^{-k}\right), 0 \leq k \leq l\right\} .
$$


The scope of the preceding results can be enlarged considerably, if one also considers transformations, which leave the form of the operators invariant. The most useful of these is the Kummer-Liouville (KL) transformation. It is based on

$$
y(x)=\mu(x) z(t), \quad t^{\prime}(x)=\gamma(x)>0 .
$$

The $(\gamma, \mu)$-KL transform maps

$$
\begin{aligned}
& p_{k} \longrightarrow p_{k} \mu^{2} \gamma^{2 k-1}+\cdots=\tilde{p}_{k}+\cdots, \\
& q_{j} \longrightarrow q_{j} \mu^{2} \gamma^{2 j-2}+\cdots=\tilde{q}_{j}+\cdots, \\
& \tilde{w}=w \mu^{2} \gamma^{-1} .
\end{aligned}
$$

Here, of course, the $\tilde{p}_{j}$ and $\tilde{q}_{j}$ are just the leading parts if the transformation is sufficiently smooth [7].

For Sturm-Liouville equations $L y=-y^{\prime \prime}+p_{0} y$ one knows that the spectrum is discrete if $p_{0}(x) \rightarrow \infty$. The extension to higher order operators of degree $2 n$ is as follows.

Assume $p_{0} / w \rightarrow \infty$. Apply a KL transform which standardizes $p_{n, s}$ and $p_{0, s}$, i.e. $\gamma=\left(\frac{p_{0 s}}{p_{n s}}\right)^{\frac{1}{2 n}}$ and $p_{0, s} \mu^{2}=\gamma$; see [7]. Assume the transformed coefficients satisfy (1.3) and that the Fourier polynomial $\tilde{\mathfrak{P}}$ based on the transformed coefficients satisfies

$$
\liminf _{x \rightarrow \infty}\left(\min _{\lambda} \tilde{\mathfrak{P}}(\lambda, x, 0)\right)>0 .
$$

Then $H$ is bounded below and has a compact resolvent. In particular $H$ has a discrete spectrum.

Note that no dichotomy condition is required for $\widetilde{\mathfrak{P}}$, because the $z$-uniform dichotomy condition is only needed for real roots of $\tilde{\mathfrak{P}}$. By assumption, however, $\tilde{\mathfrak{P}}$ has no real roots. It is fairly obvious that this result can easily be generalized to more general classes of coefficients. Sturm-Liouville operators have all of $\mathbb{R}$ as absolutely continuous spectrum if $p_{0}$ tends to $-\infty$, but not faster than $-x^{2}$. As an extension consider the following example.

Example 6. Consider an even order operator (1.1) with coefficients of approximate power type with $l=3$. Assume

$$
p_{n}=w=1
$$

and

$$
p_{0}=-p_{00}\left(1-h_{0}\right) x^{\beta}, \quad p_{00}>0, \quad \beta>0 .
$$

Then

$$
\alpha_{\gamma}=\frac{\beta}{2 n} \quad \text { and } \quad \alpha_{\mu^{2}}=-\beta\left(1-\frac{1}{2} n\right)
$$


Moreover, assume that the transformed intermediate coefficients vanish asymptotically, i.e.

$$
\alpha_{p_{k}}-\beta+\beta \frac{2 k}{2 n}, \quad \alpha_{q j}-\beta+\beta \frac{2 j-1}{2 n}<0, \quad k=1, \ldots, n-1, j=1, \ldots, n .
$$

Then

$$
\sigma_{\mathrm{ac}}(H, 1)=\mathbb{R}
$$

if the transformed weight function $x \frac{-\beta(2 n-1)}{2 n}$ is not integrable, i.e. $\beta<\frac{2 n}{(2 n-1)}$. If this term is integrable the spectrum is discrete and $\operatorname{def} T=(2 n, 2 n)$.

Proof. The characteristic Fourier polynomial is $\lambda^{2 n}-1=0$ and only the root $\lambda=1$ will generate absolutely continuous spectrum.

It is quite obvious that this example can be extended in many ways to more general coefficients. For $n=1$ we obtain the well known result that Sturm-Liouville operators are limit circle if the potential is more singular at infinity than $-c x^{2}$. The quantum mechanical interpretation of this is that in this case a particle could escape to infinity in finite time.

For the following example a transformation to a bounded coefficient form is likewise needed.

Example 7. The Everitt-Markett $6^{\text {th }}$ order Bessel type equation on $[0, \infty)$, see [16], as corrected in [15], is

$$
L(y)=w^{-1}\left\{-\left(p_{3} y^{\prime \prime \prime}\right)^{\prime \prime \prime}+\left(p_{2} y^{\prime \prime}\right)^{\prime \prime}-\left(p_{1} y^{\prime}\right)^{\prime}+p_{0}\right\} y
$$

with $w=p_{3}=x^{3}, p_{2}=33 x, p_{1}=225 / x-96 / M x^{3}, M>0$ and $p_{0}=0$. At first we consider only the problem on $[1, \infty)$ and perform a standard KL transform. With $\mu=x^{-\frac{3}{2}}$ and $\gamma=1$ we get for the transformed coefficients: $w=1, \widetilde{p}_{3}=1, \widetilde{p}_{2}=$ $c_{2} / x^{2}, \tilde{p}_{1}=-96 / M+c_{1} / x^{4}, \tilde{p}_{0}=c_{0} / x^{2}+c_{0}^{\prime} / x^{6}$ where the $c_{i}$ are constants. Thus the theory above can be applied. Now the absolutely continuous spectrum can be read off from the graph of the limiting Fourier polynomial,

$$
Q(\lambda)=\lambda^{6}-\frac{96}{M} \lambda^{2} .
$$

Thus $L$ restricted to $[1, \infty)$ has no singular continuous spectrum and

$$
\sigma_{\mathrm{ac}}(H, 2)=\left[-\left(\frac{32}{M}\right)^{\frac{3}{2}}(\sqrt{3}-1), 0\right), \quad \sigma_{\mathrm{ac}}(H, 1)=[0, \infty) .
$$

For the equation restricted to $(0,1]$ we use the Frobenius theory, because 0 is a weakly singular point. The indicial equation is

$$
\rho^{2}(\rho-1)^{2}+33 \rho(\rho-1)^{2}(\rho-2) 225 \rho(\rho-2)=0 .
$$


The roots are (see also [16]) $\rho=0,2,6,4,-4,-2$. Thus there are four uniformly square integrable solutions and the deficiency index of the Everitt-Markett Bessel operator is $(4,4)$. By Theorem 3.1 applied to the transformed operator on $[1, \infty)$ and the decomposition method, $H$ has no singular continuous spectrum. The corresponding fourth order Bessel equation is

$$
\left(x y^{\prime \prime}\right)^{\prime \prime}-\left(\left(\frac{q}{x}+\frac{8}{M} x\right) y^{\prime}\right)^{\prime}=z x y, \quad x>0, M>0 .
$$

A transformation as above with $\gamma=1$ and $\mu^{2}=x^{-1}$ leads to $\tilde{p}_{2}=\tilde{w}=1$ and $\tilde{p}_{1}=p_{1} x^{-1}-1 / x^{2}$. Thus the limiting polynomial is $\lambda^{4}+8 / M \lambda^{2}=z$ which gives rise to $\sigma_{\mathrm{ac}}(H, 1)=[0, \infty)$ for the operator restricted to $[1, \infty)$. The indicial equation is $\rho(\rho-2)\left((\rho-1)^{2}-9\right)=0$ and thus def $T=(3,3)$.

Above we had always assumed that the conditions for asymptotic integration automatically hold for all of $\mathbb{R} \backslash \mathscr{E}$ where $\mathcal{E}$ is discrete. The following example shows that this need not be the case.

Example $8\left(L y=(-1)^{n} y^{(2 n)}+\left(A \cos x^{\alpha}\right) y, A>0,0<\alpha<1\right)$. For real $z$ with $|z|>A$ the corresponding system can be transformed into Levinson form in two steps if $\alpha<1 / 2$. For $1>\alpha \geq 1 / 2$ more steps are needed. Then our methods give $\sigma_{\mathrm{ac}}(H, 1) \supset(A, \infty)$. Since $T \geq-A$ there can at most be $n$ bound states below $-A$. It is now easy to construct for any $z \in(-A, A)$ approximate $z$ eigenfunctions located in large $x$-domains where $\left|A \cos x^{\alpha}-z\right|$ is small. Thus $[-A, A] \in \sigma_{\text {ess }}(H)$. Most likely the spectrum is singular continuous or dense discrete in $[-A, A]$. For obvious reasons asymptotic integration as used above cannot be applied for $|\operatorname{Re} z|<A$.

More generally one can show for operators of the type (1.1) or (1.2) with (1.3) replaced by

$$
f=f_{1}+f_{2}+f_{3} \text { and } f_{1} \text { bounded, } f_{1}^{\prime 2}, f_{1}^{\prime \prime}, f_{2}^{\prime}, f_{3} \in \mathscr{L}^{1},
$$

the existence of a constant $K>0$ with

$$
\begin{aligned}
& {[K, \infty) \subset \sigma_{\mathrm{ac}}(H, 1) \text { for even order operators on }[0, \infty),} \\
& (-\infty,-K] \cup(K, \infty) \subset \sigma_{\mathrm{ac}}(H, 1) \text { for odd order operators in } \mathbb{R} .
\end{aligned}
$$

Physicists have always assumed that the essential spectrum on the half line for Sturm-Liouville operators is automatically absolutely continuous. Likewise they assume that bound state energies have to be negative. So the example of Wigner and von Neumann, which leads to a bound state embedded in the continuum came as a real surprise. The higher order analog are operators (1.1) or (1.2) with $p_{0}=a x^{-\beta} \sin x$ and the only other non-trivial coefficient $p_{n}=1$ or $q_{n+1}=1$.

Example 9 (Wigner-von Neumann potential). In the even order case the operator amounts to

$$
L y=(-1)^{n} y^{(2 n)}-p_{0} y-z y \quad p_{0}=a x^{-\alpha} \sin x, 1 \geq \alpha>2 / 3 .
$$


We will treat $p_{0}$ as a perturbing term and diagonalize the main part of the corresponding system. The roots of the characteristic Fourier polynomial are the $2 n$ roots $\lambda_{k}=|z|^{1 / 2 n} e^{i \beta / 2 n+2 \pi k i / 2 n}$ with $\beta=0$ if $z>0$ and $\beta=\pi$ if $z<0$. The $k$-th eigenvector is then [3] with $\mu_{k}=i \lambda_{k}$ the $k$-th root (2.17),

$$
w_{k}=\left(2 n \mu_{k}^{2 k-1}\right)^{-1 / 2}\left(1, \mu_{k}, \ldots, \mu_{k}^{n-1},(-1)^{n-1} \mu_{k}^{(2 n-1)}, \ldots, \mu_{k}^{n}\right),
$$

and the $T=\left(w_{1}, \ldots, w_{2 n}\right)$ transformed system $T v=u$ has the form

$$
v^{\prime}=\left(\operatorname{diag}\left(\mu_{j}\right)+\left(T^{-1} \bigodot_{4} T\right)\right) v=(\Lambda+S) v .
$$

The only nontrivial matrix element of $\boldsymbol{C}_{4}$ is $\left(\boldsymbol{C}_{4}\right)_{1, n+1}=p_{0}$. With [3], eq. (3.13), we get $\left(T^{-1} \bigodot_{4} T\right)_{j k}=-(2 n)^{-1} \mu_{k}^{(2 n-1) / 2} \mu_{j}^{(2 n-1) / 2} p_{0}=S_{j k}$. To (3.17) apply a $(1+\mathrm{Q})$-transform, $(1+Q) v_{1}=v$ with $Q \rightarrow 0$. Then

$$
v_{1}^{\prime}=\left[\Lambda+(1+Q)^{-1}\left(-Q^{\prime}+\Lambda Q-Q \Lambda+\digamma_{4}+\iota_{4} Q\right)\right] v_{1} .
$$

We are looking for a matrix $Q$ with $Q \rightarrow 0$ and $Q_{i j}^{\prime}=\left(\Lambda Q-Q \Lambda+C_{4}\right)_{i j}$,

$$
Q_{j k}^{\prime}=\left(\mu_{j}-\mu_{k}\right) Q_{j k}+S_{j k}
$$

If $\operatorname{Re}\left(\mu_{j}-\mu_{k}\right) \neq 0$ a $Q_{j k}$ with $Q_{j k}=O\left(x^{-\alpha}\right)$ can be found. For such index pairs the corresponding contribution of $Q \mathcal{C}_{4}$ will be integrable and may be neglected by Levinson's theorem. Now $\operatorname{Re} \mu_{k}=\operatorname{Re} \mu_{j}$ implies $\sin \left(\frac{\beta}{2 n}+\frac{2 \pi k}{2 n}\right)=$ $\sin \left(\frac{\beta}{2 n}+\frac{2 \pi j}{2 n}\right)$. Assume $k<j, n$ then $n-\beta / \pi=(j+k)$ or $3 n-\beta / \pi=(j+k)$. Even if $\operatorname{Re}\left(\mu_{j}-\mu_{k}\right)=0$, equation (3.18) will have a solution $Q_{j k}=O\left(x^{-\alpha}\right)$ if $\operatorname{Im}\left(\mu_{j}-\mu_{k}\right) \neq \pm 1$, the frequency of the sine. For simplicity assume now $z>0$ or $\beta=0$. Then we have resonance between the sine and $\mu_{j}-\mu_{k}$ if

$$
2 \cos \frac{2 \pi}{2 n} j \cdot|z|^{1 / 2 n}= \pm 1
$$

For each $z>0$ there are therefore at most two possible resonance pairs. If $z$ is nonresonant the matrix $Q$ can be constructed for all pairs of indices and all solutions of (3.16) look approximately like the solutions of the unperturbed system. Now assume that $z>0$ is resonant. Then the corresponding two dimensional resonant subsystem has the form

$$
u^{\prime}=\left(\begin{array}{cc}
\mu_{k} & b p_{0} \\
b p_{0} & \mu_{j}
\end{array}\right) u
$$

with

$$
\begin{aligned}
\mu_{k} & = \pm \frac{i}{2}-z^{1 / 2 n} \sin \frac{2 \pi}{2 n} k \\
b & =\frac{-1}{2 n} z^{\frac{2 n-1}{2 n}} e^{i \pi(2 n-1) / 2},
\end{aligned}
$$


and

$$
\mu_{j}=\mp i-z^{1 / 2 n} \sin \frac{2 \pi}{2 n} k
$$

for $0<k<n$. Factoring out $\operatorname{diag}\left(\exp \mu_{k} t, \exp \mu_{j} t\right)$, we finally reduce (3.20) to

$$
v^{\prime}=\left(\begin{array}{cc}
0 & c_{1} \\
c_{2} & 0
\end{array}\right) v, \quad c_{i}=e^{ \pm 2 i x} b p_{0},
$$

which can be solved by asymptotic integration giving rise to solutions of the form

$$
\left(\begin{array}{c}
\sqrt{c_{1}} \\
\pm \sqrt{c_{2}}
\end{array}\right)(1+o(1)) \exp \left( \pm \int_{a}^{x} \sqrt{c_{1} c_{2}} d t\right)
$$

Thus in this case (3.20) has two square integrable solutions, which decay at infinity exponentially. Whether these are proper eigenfunctions, depends of course on the boundary condition. The case $n<k<j$ leads to exponentially increasing eigenfunctions.

A particular role is played by $k=0, j=n$, because in this case the exponential factor in (3.20) vanishes and the solutions behave like $\exp \left( \pm \int_{a}^{x} \sqrt{c_{1} c_{2}} d t\right)$. Thus for $\alpha<1$ one of them is square integrable and the other not. The case $\alpha=-1$, which gives the original Wigner-von Neumann situation, leads to solutions with power behavior and an eigenstate if the amplitude $a$ is large enough. The case $z<0$ is similar.

Summing up we have: for the Wigner-von Neumann problem (3.16) we have $\sigma_{\mathrm{ac}}(H, 1)=[0, \infty)$. There are finitely many $z$-values, $z \neq 0$, where the solutions oscillate in resonance with the sine, i.e., the frequency or energy is fixed. There are at most two square integrable solutions for a given resonance value $z$. These may give rise to eigensolutions if the boundary conditions fit. In particular a change in the boundary condition will destroy the eigenfunctions. For odd order operators we will consider

$$
(-1)^{n} i y^{(2 n+1)}+(1+|x|)^{-\alpha}(\sin x) y \text { on } \mathbb{R} \text {. }
$$

Now the sign of $z$ is irrelevant and we may assume $z>0$. The eigenvalues are $\mu_{k}=i|z|^{1 / 2 n} \exp \frac{2 \pi i}{2 k+1} k, 0 \leq k \leq 2 n$ and the same procedure as above leads again to the situation $\sin \frac{2 \pi j}{2 n+1}=\sin \frac{2 \pi k}{2 n+1}$ for $0<k<j, n$. This, however, would lead to $(2 n+1) \pi=2 \pi(j+k)$, which is impossible. Thus in this case there are no embedded bound states.

Example 10 (Rapidly oscillating potentials). In [3] it was shown that the perturbation of the coefficients $f=p_{k}, q_{j}$ by terms of the form $f_{4}$ with

$$
\hat{f}(x)=\int_{x}^{\infty} f(t) d t \in \mathscr{L}^{1}
$$


does not alter the spectral properties of the Hamiltonian $H$. For this reason consider more singular terms. Again we consider only a very simple case

$$
L y=(-1)^{n} y^{(2 n)}+(-1)^{k}\left(p_{k} y^{(k)}\right)^{(k)}
$$

with

$$
p_{k}=h(x) \sin g(x), \quad g, h \in \mathcal{C}^{2}, g^{\prime} \nearrow \infty, h \cdot g^{-1} \text { bounded. }
$$

Rewrite (3.25) in systems form (2.2) and denote the perturbing oscillatory term by $\mathcal{C}_{4},\left(\mathcal{C}_{4}\right)_{k-1, n+k-1}=p_{k}$. Apply to

$$
u^{\prime}=\left(e+e_{4}\right) u
$$

a simple $(1+Q)$-transform, i.e. let $Q_{k-1, n+k-1}=\hat{p}_{k}$ with $\hat{p}_{k}^{\prime}=p_{k}$, and let $(1+Q) v=u$. Then

$$
v^{\prime}=(\mathcal{C}+(1-Q)(\mathcal{Q}-Q \mathcal{C})) v .
$$

Assume now $0 \leq k<n-1$ and

$$
\left(\frac{h}{g^{\prime}}\right)^{\prime \prime}\left(g^{\prime}\right)^{-2},\left(\left(g^{\prime}\right)^{-1}\right)^{\prime} \in \mathscr{L}^{1}, \quad\left(\frac{h}{g^{\prime}}\right),\left(\frac{h}{g^{\prime}}\right)^{\prime} \text { bounded. }
$$

Then two $(1+Q)$-transformations turn the oscillatory terms into Levinson terms. For $k=n-1$, however, a term $-\hat{p}_{n-1}^{2}$ arises in the position of $p_{n-1}$. All other terms can be removed by further $(1+Q)$-transformations. Now we have

$$
\hat{p}_{k}=-\frac{h}{g^{\prime}} \cos g+\left(\frac{h}{g^{\prime}}\right)^{\prime} \frac{1}{g^{\prime}} \sin g \bmod \mathscr{L}^{1} .
$$

The nonoscillatory part of $\hat{p}_{k}^{2}$ is $\frac{1}{2}\left(\frac{h}{g^{\prime}}\right)^{2}$. Thus if $h=a g^{\prime}$ the characteristic polynomial is altered to

$$
\mathfrak{P}=\lambda^{2 n}-\frac{1}{2} a^{2} \lambda^{2(n-1)}-z .
$$

This leads to a different absolutely continuous spectrum. In this case we have

$$
\sigma_{\mathrm{ac}}(H, 2)=\left[-\frac{1}{n} \frac{a^{2}}{2}\left(\frac{1}{2} a^{2} \frac{n-1}{n}\right)^{2 n-1}, 0\right] \quad \text { and } \quad \sigma_{\mathrm{ac}}(H, 1)=[0, \infty) .
$$

Now consider a perturbation by an odd order term $q_{j}, j<n-1, q_{j}=h \sin g$. Then $B C_{4}=B Q_{4}=0$ and two $(1+\mathrm{Q})$-transformations effectively remove all oscillatory terms if (3.27) holds. A term $q_{n-1}=h \sin g$ leads to a nonoscillatory term $\frac{1}{2}\left(\frac{h}{g^{\prime}}\right)^{2}$ in the $p_{n-1}$-position. A perturbation by an oscillatory $q_{n}$ term plays a particular role, because the $q_{n}$ term appears in the diagonal of $\mathcal{C}$ (2.2), $\ell_{n n}=-q_{n}^{2}=-h^{2} \sin ^{2} g=-\frac{1}{2} h^{2}+\frac{1}{2} h \cos 2 g$ and $A_{n n}=-i q_{n}$. In this case the oscillatory terms can be removed by two (1+Q)-transformations if $\frac{h}{g^{\prime}} \in \mathscr{L}^{2}$ so that only the non-oscillatory term $-\frac{1}{2} h^{2}$ remains in the position of $p_{n-1}$. 
Example 11. As a final example consider the perturbation of odd order operators by a highly oscillatory term. Thus we are looking at

$$
L y=(-1) i y^{(2 n+1)}+i(-1)^{k+1}\left(\left(q_{k} y^{(k)}\right)^{k-1}+\left(q_{k} y^{(k-1)}\right)^{(k)}\right) .
$$

With $k \leq n-1$ and $q_{k}=h \sin g$ as above the (1+Q)-transforms will convert the oscillatory terms into Levinson terms if the conditions (3.27) hold. A perturbation by $q_{n}$ of the form (3.26) leads after one $(1+Q)$-transform to a nonoscillatory term $\frac{1}{2}\left(\frac{h}{g^{\prime}}\right)^{2}$. All other terms can be removed effectively by further $(1+Q)$-transform. thus in this case the situation is identical with that of example 10. In particular a term with $h=a g^{\prime}$ can be used to modify the spectrum.

Remark 3.3. These examples exhibit the inertia exerted by differential equations. Generally solutions will ignore rapid oscillations. Only if their amplitude $h$ exceeds the frequency $\left(g^{\prime}\right)^{-1}$ will one have an observable effect. The effect itself is quadratic and results from the interaction of the solution - first $(1+Q)$-transform - with the potential $p_{n-1}$ respectively $q_{n}$. Lower order perturbations will have no effect.

\section{Operators with unbounded coefficients}

With unbounded coefficients a number of things change in the analysis of higher order operators. Now it is not any more possible to estimate lower order terms against higher order ones. For this reason the analysis of higher order operators with unbounded coefficients has been restricted mostly to operators with few terms. On the other hand new and interesting phenomena arise from competing terms. The most obvious of these effects is the change in the deficiency index. However, even there the emphasis has been to construct examples which demonstrate the range of the deficiency index or the existence of non limit point operators with essential spectrum For asymptotic integration unbounded coefficients do not any more allow to enforce the dichotomy condition via the spectral parameter. Thus it has to be postulated in general. Secondly the remainder term in Levinson's theorem competes with small spectral values or is multiplied by large terms, so that estimates are difficult to come by. Chapter $3.8 \mathrm{ff}$ in Eastham's book shows clearly some of the problems arising. Finally the back transformation may be problematic. A number of these problems have been solved recently by more precise estimates of the remainder [11]. Though $z$-uniform estimates still present a formidable problem.

For this reason we begin with a rather general class of examples for which the difficulties in asymptotic integration are minimal and which nonetheless exhibit a wide range of phenomena.

4.1. Power class operators. One of the main obstacles in the understanding of higher order differential operators is our lack of understanding and control of higher 
order polynomials. For this reason we will introduce here a class of differential operators with power coefficients where these problems are minimized. Of course operators with power coefficients have been studied before, but never in a systematic fashion with the spectral theory in view. Previously this class has been introduced in [11]. Our starting point in the characteristic Fourier polynomial

$$
\mathfrak{P}(x, \lambda, z)=\sum_{0}^{m} r_{k}(x) \lambda^{k}-z w=\sum_{0}^{m} c_{k} x^{\alpha_{k}} \lambda^{k}-z w, \quad c_{m}=1 .
$$

To translate $\mathfrak{P}$ into an operator on $[1, \infty)$ let $p_{k}=r_{2 k}$ and $q_{j}=r_{2 j-1}$. Thus we handle odd and even operators simultaneously. Our class of examples is based on the concept of pivot coefficients $k_{i}$.

$$
m=k_{l}>k_{l-1}>\ldots>k_{1}>k_{0}=0, \quad c_{k_{j}} \neq 0, j=1, \ldots, l .
$$

The idea is to assign to the $j$-th interval $I_{j}=\left\{k_{j-1}, \ldots, k_{j}\right\}$ a polynomial $\mathfrak{P}_{j}$, the $j$-th segmental polynomial,

$$
\begin{aligned}
\mathfrak{P}_{j}(x, \lambda) & =\sum_{k \in I_{j}} c_{k} x^{\alpha_{k}} \lambda^{k} \\
& =\sum_{k \in I_{j}}\left(c_{k} x^{\alpha_{k}-\alpha_{\alpha_{j-1}}} \lambda^{k-k_{j-1}}\right) x^{\alpha_{k_{j-1}}} \lambda^{k_{j-1}} \\
& =\tilde{\mathfrak{P}}_{j} x^{\alpha_{k_{j-1}}} \lambda^{k_{j-1}}
\end{aligned}
$$

so that the reduced segmental polynomial $\tilde{\mathfrak{P}}_{j}$ has roots $\lambda$ of the form $\mu x^{\beta_{j}}=\lambda(x)$ with $\mu$ a root of $\hat{\mathfrak{P}}_{j}=\sum_{k \in I_{j}} c_{k} \mu^{k-k_{j-1}}$. This requires

$$
\alpha_{k}=\alpha_{k_{j-1}}-\left(k-k_{j-1}\right) \beta_{j}
$$

In addition the roots should be sufficiently distinct. Therefore we demand

$$
\beta_{l}>\beta_{l-1}>\beta_{l-2}>\ldots>\beta_{1} \text { and } w=x^{\beta_{1}} \text { or } w^{\beta_{1}-\varepsilon}, \quad \varepsilon>0 .
$$

It follows from (4.4) that $\alpha_{k_{j}}=\alpha_{k_{j-1}}-\left(k_{j}-k_{j-1}\right) \beta_{j}$ and thus

$$
\alpha_{k_{j}}=-\left(k_{j}-k_{j-1}\right) \beta_{j}-\left(k_{j-1}-k_{j-2}\right) \beta_{j-1}-\cdots-\left(k_{1}-k_{0}\right) \beta_{1}+\alpha_{0} .
$$

For the repeated diagonalizations and for the nonintegrability of the coefficients we also need

$$
\alpha_{0}, \beta_{1}>-1 \text {. }
$$

The typical size of an eigenvalue from segment $j$ is thus $x^{\beta_{j}}$, so that its contribution at the $k$-th element is $c_{k} x^{\alpha_{k}} \cdot x^{k \beta_{j}}$. For $k \in I_{j}$ this is by (4.4)

$$
c_{k} x^{\alpha_{k_{j-1}}-\left(k-k_{j-1}\right) \beta_{j}} \cdot x^{\left(k-k_{j-1}\right) \beta_{j}+k_{j-1} \beta_{j}} .
$$


Apart from the factor $c_{k}$ this is independent of the index $k \in I_{j}$. Since $c_{k_{j}} \neq 0$ all roots of $\tilde{\mathfrak{P}}_{j}$ are different from 0 . A root $\lambda=\mu x^{\beta_{j}}$ evaluated at some term $r_{k} \lambda^{k}$ for some $k \notin I_{j}$ contributes at most the $x^{-\delta}$ part of the $I_{j}$ contribution, where $\delta=\min \left(\beta_{j+1}-\beta_{j}\right)$. To see this it suffices to consider neighboring segments only and to evaluate the expressions at the bordering pivot coefficients. See also [11]. Now write the characteristic Fourier polynomial $\mathfrak{P}$ as

$$
\mathfrak{P}(x, \lambda, z)=\mathfrak{P}_{j}(x, \lambda, z)+\mathfrak{R}_{j}(x, \lambda, z),
$$

and let $\lambda=\mu x^{\beta_{j}}$ be a root of $\mathfrak{P}_{j}$. Then, as we have seen $\mathfrak{R}\left(x, \mu x^{\beta_{j}}, z\right)=o\left(x^{-\delta}\right)$. Thus a $\mathfrak{P}_{j}$ root is asymptotically almost a root of $\mathfrak{P}$. Standard perturbation theory now shows that there is a root $\lambda^{\prime}$ of $\mathfrak{P}$ with $\left|\lambda(x)-\lambda^{\prime}(x)\right|=o\left(x^{-\delta}\right)$. If all roots of class $j$ are distinct these roots give rise to $\left(k_{j}-k_{j-1}\right)$ distinct roots of $\mathfrak{P}$. Thus if all segmental polynomials have distinct roots, this exhausts all roots of $\mathfrak{P}$. Summing up we have shown the following result.

Proposition 4.1. Let $\mathfrak{P}$ be a polynomial with real power coefficients satisfying (4.2), (4.5) and (4.6). Moreover assume that all segmental polynomials $\mathfrak{P}_{j}$ have distinct roots. Then to each root $\lambda=\lambda(x, z)$ of $\mathfrak{P}$ there corresponds a unique segment $j$ and root $\tilde{\lambda}$ of the segmental polynomial $\mathfrak{P}_{j}$, the shadow root, with

$$
|\lambda(x, z)-\tilde{\lambda}(x, z)|=o\left(x^{-\delta}\right) .
$$

Proof. It is easy to see that all these terms differ at most in their factors $c_{k}$. It has been shown in [11], Lemma 3.2, that for $k \notin I_{j}$,

$$
\left(r_{k} x^{k \beta_{j}}\right)\left(r_{k_{j}} x^{k_{j} \beta_{j}}\right)^{-1}=O\left(x^{-\delta}\right), \quad \delta=\min \left(\beta_{i}-\beta_{i-1}\right) .
$$

This implies that the $\mathfrak{P}_{j}$-eigenvalues contribute essentially to terms in $I_{j}$ and that the contribution from the remaining terms is at best $O\left(x^{-\delta}\right)$. Straight forward perturbation theory implies then that to any root of $\mathfrak{P}_{j}$ there is a corresponding root of $\mathfrak{P}$. Since this holds for any segment, and since all these segmental roots account for $\left(k_{l}-k_{l-1}\right)+\left(k_{l-1}-k_{l-2}\right)+\cdots+\left(k_{1}-k_{0}\right)=m$ roots, we can turn the argument around.

Since we have to deal with eigenvalues for $z$ values slightly off the real axis, we will need estimates for $\partial_{\lambda} \mathfrak{P}(\lambda)$, because

$$
\lambda_{j}(x, z+i \eta) \approx \lambda_{j}(x, z)+i \eta w\left(\partial_{\lambda} \mathfrak{P}\left(x, \lambda_{j}(z)\right)\right)^{-1} .
$$

Since eigenvalues $\lambda$ with $i$-shadow eigenvalues $\tilde{\lambda}$ attain their essential values at cluster $j$, it is not surprising that in this case $\partial_{\lambda} \mathfrak{P}\left(x, \lambda_{j}, z\right)$ can be estimated by

$$
\left|\left(\partial_{\lambda} \mathfrak{P}\right)(x, \lambda, z)\right|=O\left(M_{j}\right), \quad M_{j}=x^{\alpha_{k_{j}}+\beta_{j}\left(k_{j}-1\right)} .
$$


Moreover, one has

$$
M_{j-1}=O\left(x^{-\delta} M_{j}\right), \quad j=l, \ldots, 1
$$

These results reduce the determination and analysis of the roots of $\mathfrak{P}$ to that of the segmental polynomials. It remains to check the $z$-uniform dichotomy conditions. As noted before it suffices to check it only for the eigenvalues $\lambda(x, z+i \eta)$, for which $\lambda(x, z)$ is real, $z \in \mathcal{K}_{\varepsilon} \cap \mathbb{R}$. Those we will call essentially real. We can now state the dichotomy conditions needed for our analysis.

D1 The eigenvalues of each segmental polynomial are distinct and the essentially real eigenvalues $\lambda_{i}$ and $\lambda_{j}$ of segment $k$ satisfy

$$
\partial_{\lambda} \tilde{\mathfrak{P}}_{k}\left(\lambda_{i}\right) \neq \partial_{\lambda} \tilde{\mathfrak{P}}_{k}\left(\lambda_{j}\right) \neq 0 \text {. }
$$

D2 $\operatorname{sign} \operatorname{Im}\left(\lambda_{i}(x)-\lambda_{j}(x)\right)$ and $\operatorname{sign} \operatorname{Im} \lambda_{i}(x)$ is constant mod $\mathscr{L}^{1}$ for each cluster and eigenvalues $\lambda_{i}, \lambda_{j}$ associated to it. If $w=o\left(p_{0}\right)$ these conditions are not required for the lowest segment.

Once these conditions are met the eigenfunctions are given by (2.21):

$$
u_{k}(x, z)=T(1+B)\left(e_{k}+r_{k}(x, z)\right) \exp \left(\int_{a}^{x} i \lambda_{k}(t, z) d t\right) .
$$

Here $T_{1 l}=M_{l}^{-\frac{1}{2}}$, and $B$ arises from the second, third, ...diagonalization while $r_{k}$ is the remainder term from the last asymptotic integration. We will call the term $T(1+B)\left(e_{k}+r_{k}\right)$ the form factor. In order to avoid interference between the growth of the form factor and the exponential we will demand

$$
w M_{j} \geq x^{-1+\varepsilon} \quad \text { or } \quad w M_{j} \leq x^{-1-\varepsilon} \text { for } \varepsilon>0 .
$$

In order to analyze this with respect to the deficiency index and spectral properties, assume that $\lambda_{k}$ has a shadow eigenvalue in segment $j$. Then the factor $M(\lambda)_{k}^{-\frac{1}{2}}$ may be replaced by $M_{j}^{-\frac{1}{2}}$. Let $\alpha_{1}(x, z) \pm i \beta_{1}(x, z), \ldots, \alpha_{r}(x, z) \pm \beta_{r}(x, z), \gamma_{2 r+1}(x, z)$, $\ldots, \gamma_{l}(x, z)$ with $l=k_{j}-k_{j-1}$ and $\alpha, \beta, \gamma$ real be the eigenvalues of $\mathfrak{P}$ corresponding to all eigenvalues of $\tilde{\mathfrak{P}}_{j}$. Then the eigenvalues $\alpha_{m}(x, z)+i \beta_{m}(x, z), m=1, \ldots, r$ correspond to $z$-uniformly square integrable eigenfunctions. They contribute $(r, r)$ to the deficiency index and otherwise only to the discrete spectrum. For the essentially real eigenvalues write for $z \in \mathcal{K}_{\varepsilon} \cap \mathbb{R}$ as in (4.9),

$$
\gamma(x, z+i \eta) \approx \gamma(x, z)+i \eta w\left(\partial_{\lambda} \mathfrak{P}(\gamma)\right)^{-1} .
$$


Thus the exponential factor determines an exponentially decreasing eigenfunction if

$$
w\left(\partial_{\lambda} \mathfrak{P}(\gamma, x)\right)^{-1}>0,
$$

and if $w \partial_{\lambda} \mathfrak{P}^{-1}$ is not integrable. In particular if

$$
\left|w^{-1} \partial_{\lambda} \mathfrak{P}(\gamma, x)\right| \geq c x^{1-\varepsilon} \quad \text { for some } c>0, \varepsilon>0,
$$

no logarithmic terms will arise in the exponents and (4.12) defines a square integrable eigenfunctions, which loses its square integrability if $\eta \rightarrow 0$. These are the eigenfunctions that contribute to the absolutely continuous spectrum. They contribute to the deficiency index if $\partial_{\lambda} \mathfrak{P}(x, \gamma, z)>0$. The segments contribute individually to the deficiency index

$$
\operatorname{def} T=\sum_{j=1}^{l}(\operatorname{def} T)_{j},
$$

and if $w M_{j}^{-1}$ is not integrable one gets

$$
(\operatorname{def} T)_{j}= \begin{cases}\left(\frac{l}{2}, \frac{l}{2}\right) & \text { if } l=k_{j}-k_{j-1} \text { is even and } c_{k}>0 \\ & \text { or } l \text { is odd and } c_{k}<0, \\ \left(\frac{l+1}{2}, \frac{l-1}{2}\right) & \text { if } l \text { is even and } c_{k}<0 \\ & \text { or } l \text { is odd and } c_{k}>0 .\end{cases}
$$

The most problematic case arises if $w \partial_{\lambda} \mathfrak{P}(x, \gamma)^{-1}=O\left(w M_{j}^{-1}\right)$ is integrable. In this case the square integrability of $u$ (4.12) is independent of $z$ and depends entirely on the first factor $T(1+B)\left(e_{k}+r_{k}\right)$. Of these $T e_{k}=M_{k}^{-\frac{1}{2}}$ is again $w$-square integrable, but in principle nothing is known about the square integrability of

$$
M_{l}^{-\frac{1}{2}} B_{l k} \quad \text { or } \quad M_{l}^{-\frac{1}{2}}\left(r_{k}\right)_{l} \text {. }
$$

For very general coefficients this represents a rather serious problem. Fortunately enough one can show in our situation the following result.

Lemma 4.2. If $k \in I_{j}$ and if $w M_{j}^{-1}$ is integrable, then $M_{l}^{-\frac{1}{2}} B_{l k}$ and $M_{l}^{-1}\left(r_{k}\right)_{l}$ are square integrable. In this case the deficiency index $(\operatorname{def} T)_{j}$ is given by $(l-r, l-r)$.

Remark 4.3. In particular the last term requires sharp $z$-uniform estimates of the remainder.

The proof for this Lemma depends on a detailed analysis of the remainder terms in asymptotic integration [11]. 
Thus the solution $u_{k}$, see (4.12), is $z$-uniformly square integrable if $\lambda_{k}$ is not essentially real with $\operatorname{Im} \lambda_{k}>0$ or if $w M_{j}^{-1}$ is integrable. If $w M_{j}^{-1}$ is not integrable the eigenfunctions $u_{k}\left(x, z_{0}+i \eta\right)$ are square integrable as long as $\eta>0$ and

$$
\lambda_{k}\left(x, z_{0}+i \eta\right) \approx \gamma\left(x, z_{0}\right)+i M(\gamma)^{-1} w \eta, \quad M(\gamma)>0 .
$$

Such eigenfunctions which lose their square integrability will be called LSI eigenfunctions. They are generally responsible for the absolutely continuous spectrum. Let us now summarize the results obtained so far.

Theorem 4.4. a) Let $T$ denote the minimal differential operator associated to the Fourier polynomial $\mathfrak{P}$, see (4.1), satisfying (4.2), (4.3), (4.5), (4.6), and (4.13), and D1 and $\mathrm{D} 2$. Then the segments $I_{j}$ contribute independently to the deficiency index (4.14). Let $\alpha_{1} \pm i \beta_{1}, \ldots, \alpha_{r} \pm i \beta_{r}, \gamma_{2 r+1}, \ldots, \gamma_{s}, s=k_{j}-k_{j-1}$ be the roots with shadows in segment $I_{j}$. Then

$$
(\text { def } T)_{j}= \begin{cases}(s-r, s-r) & \text { if } w M_{j}^{-1} \text { is integrable, } \\ \left(\frac{s}{2}, \frac{s}{2}\right) & \text { if } w M_{j}^{-1} \text { is not integrable and s even, } \\ \left(\frac{s+1}{2}, \frac{s-1}{2}\right) & \text { if } w M_{j}^{-1} \text { is not integrable, } s \text { is odd, and } c_{k_{j}}>0, \\ \left(\frac{s-1}{2}, \frac{s+1}{2}\right) & \text { if } w M_{j}^{-1} \text { is not integrable, } s \text { is odd, and } c_{k_{j}}<0 .\end{cases}
$$

b) There exists a critical index $j_{0}$ so that $w M_{j}^{-1}$ is integrable for $j>j_{0}$.

c) If the deficiency indices are equal, then the multiplicity of the absolutely continuous spectrum is given by the number of LSI eigenfunctions from all classes. Then LSI eigenfunctions from segments $I_{j}, j \geq 2$ will generate $\mathbb{R}$ as absolutely continuous spectrum. For the lowest class $I_{1}$ the results of [3] apply.

Proof. a) Equation (4.14) follows from the fact that the square integrability of a $\lambda$-eigenfunction is determined by the $\lambda$-shadow. Now if $w M_{j}^{-1}$ is integrable and if (4.13) holds all essentially real eigenvalues lead to (uniformly square integrable) USI-eigenfunctions, while this holds only for half of the others. If $w M_{j}^{-1}$ is nonintegrable half of the non-essentially real eigenvalues give rise to USI-eigenfunctions. An essentially real eigenvalue $\gamma$ gives rise to an LSI-eigenfunction if $w M_{j}^{-1}$ is not integrable and if $w \partial_{\lambda} \mathfrak{P}(x, \gamma)>0$. This, however, holds if $\partial_{\lambda} \mathfrak{P}_{j}(x, \tilde{\gamma})>0$, where $\tilde{\gamma}$ is the shadow eigenvalue of $\gamma$. This reduces the analysis to $\widehat{\mathfrak{P}}_{j}$.

b) Follows from the fact that $M_{j} \geq M_{j-1}$. To see this evaluate $M_{j}$ at $k_{j-1}$. We get $M_{j}=x^{\alpha_{k_{j-1}}+\left(k_{j-1}-1\right) \beta_{j}} \geq x^{\alpha_{k j-1}+\left(k_{j-1}-1\right) \beta_{j-1}}=M_{j-1}$.

c) It follows from the general theory [3] and [11] that only LSI eigenfunctions will contribute to the absolutely continuous spectrum. Since for all classes $j>1$, 
$\mathfrak{P}(x, \lambda, z)=\mathfrak{P}_{j}(x, \lambda)+\mathfrak{R}_{j}(x, \lambda, z)$ the eigenfunctions will not depend critically on $z$ so that in this case all of $\mathbb{R}$ arises as absolutely continuous spectrum.

Remark 4.5. It is obvious that Theorem 4.4 can be extended in many ways. The growth functions $x^{\beta_{i}}$ can be chosen fairly arbitrarily as long as the order relation (4.5) and (4.6) hold. The reduced polynomials $\tilde{\mathfrak{P}}_{j}$ could be weakly $x$-dependent and (4.13) could be abandoned at the cost of other technical conditions. However, the uniform estimates of the form factors require some form of monotonicity of the growth factors. All this, however, would not alter the underlying structure of the operators considerably. Likewise it is obvious that the power behaviour has to hold only for large $x$. This is important for operators on $\mathbb{R}$, which can be patched together smoothly near an arbitrarily chosen point.

Example 12. For even order operators of order $2 n$ all deficiency indices $(d, d), n \leq$ $d \leq 2 n$ can be realized. There are examples with $n \leq d \leq 2 n-1$ for which the absolutely continuous spectrum is nontrivial.

As an example let the pivot indices be $0<2<4<\ldots<2 n$. Let $j_{0}$ be the critical index (Theorem 4.4b). Now choose the constants $c_{2 k}$ so that all

$$
\tilde{\mathfrak{P}}_{j}=c_{2 j} x^{-2 \beta_{j}} \lambda^{2}-c_{2(j-1)}
$$

have real roots, i.e. $\operatorname{sign} c_{2 k} \neq \operatorname{sign} c_{2(k-1)}$. Then

$$
\operatorname{def} T=2\left(n-j_{0}\right)+j_{0} .
$$

If $j_{0} \geq 1$ let $\beta_{1}=0, p_{0}=0$ and $w=1$. Then

$$
\mathfrak{P}_{1}=c_{2} \lambda^{2}-z
$$

so that the contribution of the lowest class to the absolutely continuous spectrum is $[0, \infty)$ if $c_{2}>0$ and $(-\infty, 0]$ if $c_{2}<0$.

Here is a two segment example with the same result.

Example 13. Let $2 n, 2$ and 0 be the pivot indices and let $\beta_{2}=\beta>0=\beta_{1}$ be the exponents. Then

$$
\alpha_{k}=(k-2) \beta, \alpha_{1}, \alpha_{0}=0
$$

while

$$
M_{2}=x^{-(n-2) \beta+(2 n-1) \beta}=x^{(n+1) \beta}
$$

and

$$
M_{1}=1 .
$$

Assume $\beta>\frac{1}{n+1}$ then $M_{2}^{-1} w$ is integrable and

$$
\operatorname{def} T=(2 n-1-r, 2 n-1-r)
$$


where $2 r$ is the number of non-real roots of $\tilde{\mathfrak{P}}_{2}$. Again the absolutely continuous spectrum is as above. If $\beta<\frac{1}{n+1}$ we have

$$
\operatorname{def} T=(n, n)
$$

and

$$
\sigma_{\mathrm{ac}}(H, n-r)=[0, \infty)
$$

and

$$
\sigma_{\mathrm{ac}}(H, n-r-1)=(-\infty, 0)
$$

if $c_{2}>0$ and $c_{0}=0$.

The next example is again an even order operator, but which behaves almost like an odd order operator.

Example 14. Let $p_{n}=r_{2 n}=1$ and let $r_{k}=c_{k} x^{\beta k}, 0<\beta<1, k<2 n, w=1$, $c_{0}=0$. In first order the large eigenvalue $\lambda_{1}$ is given by the root of $\mathfrak{P}_{2}$,

$$
\lambda_{1} \approx-r_{2 n-1}
$$

with

$$
\operatorname{Im} \lambda_{1} \approx \operatorname{Im} z \cdot\left(\partial_{\lambda} \mathfrak{P}\left(\lambda_{1}\right)\right)^{-1} \approx \operatorname{Im} z \cdot\left(-c_{2 n-1}^{2 n-1} x^{(2 n-1)^{2} \beta}\right)^{-1} .
$$

Thus if $\beta(2 n-1)^{2}<1$ and $c_{2 n-1}>0\left(c_{2 n-1}<0\right)$, then

$$
(\operatorname{def} T)_{2}=(0,1) \quad\left((\operatorname{def} T)_{2}=(1,0)\right) .
$$

The roots of $\tilde{\mathfrak{P}}_{1}$ are of the form $\mu x^{-\beta}$ where $\mu$ is a root of

$$
\tilde{\mathfrak{P}}_{1}=c_{2 n-1} \lambda^{2 n-1}+\ldots+\left(c_{0}-z\right) .
$$

For $c_{2 n-1}>0$ this polynomial will have $2 r$ non-real roots and $(2 n-1)-2 r$ real roots. Of these $\frac{(2 n-2-2 r)}{2}+1$ satisfy

$$
\partial_{\lambda} \hat{\mathfrak{P}}_{1}(\gamma)>0 .
$$

Thus

$$
\operatorname{def} T=(\operatorname{def} T)_{2}+(\operatorname{def} T)_{1}=(0,1)+(n, n-1)=(n, n)
$$

and $T$ is limit point. This is also true for $c_{2 n-1}<0$ by the same reasoning. With the $z$-value as above the multiplicity of the absolutely continuous spectrum would be $(n-r)$. This, however, is $z$-dependent. Thus we have islands of higher $\sigma_{\mathrm{ac}}$-multiplicity in a sea of multiplicity 1 . 
Example 15. Consider a 2-segment operator with $k_{2}=2 n, k_{1}=2 k+1, w=1$ so that $M_{2}^{-1}$ is integrable and $M_{1}^{-1}$ non-integrable. Then $\mathfrak{P}_{2}$ is of odd degree. Let $\alpha_{1} \pm i \beta_{r}, \ldots, \alpha_{r} \pm i \beta_{r}, \gamma_{2 r+1}, \ldots, \gamma_{s}, s=2 n-(2 k+1)$ be the roots of $\tilde{\mathfrak{P}}_{2}$. Then

$$
(\operatorname{def} T)_{2}=(r+(s-2 r), r+(s-2 r))
$$

while

$$
(\operatorname{def} T)_{1}=(k+1, k) \quad \text { or } \quad(\operatorname{def} T)_{1}=(k, k+1)
$$

depending on the sign of $c_{2 k+1}$. Thus $T$ does not have equal deficiency indices. The lowest order for this to occur is 4 .

Odd order operators on a half line generally have unequal deficiency indices. With unbounded coefficients, however this need not be the case. Again we take a two segment example.

Example 16. For this class of examples let $m=2 n+1=k_{2}$ and $k_{1}=2 k$. Choose $w=1, \beta_{1}=0$ and choose $\beta=\beta_{2}$ so that $M_{2}^{-1}$ is integrable. Then

$$
(\operatorname{def} T)_{2}=(s, s)
$$

and

$$
(\operatorname{def} T)_{1}=(k, k)
$$

with $s=(2 n+1)-2 k-2 r$ if $\mathfrak{P}_{2}$ has $2 r$ nonreal roots. The simplest example of such an operator is 3, e.g. $r_{3}=x^{-\beta}, r_{2}=1, r_{1}=0=r_{0}, w=1$ with $\beta>1$. Then $\operatorname{def} T=(2,2)$ and $\sigma_{\mathrm{ac}}(H, 1)=[0, \infty)$.

We will not present here operators with unbounded coefficients on $\mathbb{R}$ because the decomposition theory takes care of that. The final class of examples we want to consider are a form of hybrid models. Again we consider only the simplest two segment models.

Example $17\left(k_{2}=2 n, k_{1}=2, \beta_{2}=\beta>0, \beta_{1}=0, w=1, p_{k}=c_{k} x^{-2(k-1) \beta}\right.$, $\left.1 \leq k \leq n, c_{2 n}=c_{2}=1\right)$. Only $p_{0}$ will not be of power type. We assume $p_{0}$ to be bounded, $l$-times differentiable function with $p_{0}^{(k)} \in \mathscr{L}^{\frac{l}{k}}, p_{0}^{(k)}=o(1), k \leq l$. Then with $0<\beta<\frac{1}{2(n-1)}$ we have

$$
\operatorname{def} T=(n, n)
$$

and

$$
\sigma_{\mathrm{ac}}(H)=\left(\sigma_{\mathrm{ac}}(H)\right)_{1}+\left(\sigma_{\mathrm{ac}}(H)\right)_{2}
$$

with $\left(\sigma_{\text {ac }}(H, k)\right)_{2}=\mathbb{R}$ if the segmental polynomial $\mathfrak{P}_{2}$ has $2 k$ real roots. $\left(\sigma_{\mathrm{ac}}(H)\right)_{1}$ depends only on the second order operator arising from $\mathfrak{P}_{1}$. The sum should be understood additive with respect to the multiplicities. As a special case consider again $p_{0}=A \cos x^{\alpha}, 0<\alpha<\frac{1}{2}$. As before one can now show that the spectrum is a superposition of $\sigma(H)_{2}$ and $\sigma(H)_{1}$. So even if $\sigma(H)_{2}$ is discrete we still have $\sigma_{\text {ess }}(H)=[-|A|, \infty)$ and $\sigma_{\mathrm{ac}}(H, 1)=[|A|, \infty)$. 
4.2. The singular case. For differential operators of segmental type as they were considered above or in [11] the second diagonalization by a matrix of the form $(1+B)$, with $B_{i j}=\left(\lambda_{i}-\lambda_{j}\right)^{-1}\left(T^{-1} T^{\prime}\right)_{i j}$ breaks down if $B$ does not vanish at infinity. In examples with coefficients of approximate power type one has

$$
\left(T^{-1} T^{\prime}\right)_{i j}=O\left(x^{-1}\right), \quad \text { respectively } \quad\left(T^{-1} T\right)_{i j}=O\left(x^{-1-\delta}\right),
$$

if $i$ and $j$ are from the same, respectively different, segments. In this case, which was called the singular case in [6], it is advantageous to diagonalize the subsystems matrix associated to the troublesome classes first and then proceed as before with further diagonalizations or with $(1+Q)$-transforms. In this critical case the lowest segment submatrix has the form

$$
x^{-1}\left(\operatorname{diag}\left(x \lambda_{i}\right)+R_{i j}\right)=x^{-1} D_{i j}, \quad R_{i j}=-x\left(T^{-1} T^{\prime}\right)_{i j} .
$$

The matrix $D$ is again of approximate power type and if its eigenvalues are distinct one can find a diagonalizing transformation $S_{1}$ with $x\left\|S^{-1} S^{\prime}\right\|=o(1)$. Let $S_{2}$ be a diagonalizing transformation for the higher classes then $T_{1}=S_{1} \oplus S_{2}$ will transform the system into Levinson form, because the off-segment matrix elements are $O\left(x^{-1-\delta}\right)$ and thus integrable. This form of $T_{1}$ is of course due to the decoupling of all segments in this class of operators. The eigenvalues of the lowest segment part of the systems matrix are essentially derived from the segmental polynomial $\mathfrak{P}_{1}$, because the contribution from the other classes is $O\left(x^{-1-\delta}\right)$ at most. Thus the spectral part can be computed from $\mathfrak{P}_{1}$ alone.

For the remaining discussion assume the operator to be in standard form, i.e. $p_{n}=w=1$. For $\beta_{1}<-1$ we have $w M_{1}^{-1}=x^{\beta_{l}}$. Thus all terms $w M_{j}^{-1}$, $j=2, \ldots, l$ are integrable. This shows the following result

Proposition 4.6. Assume $T$ has equal deficiency indices and $\beta_{1}<-1$. Then the spectrum of $H$ is discrete.

In this case $w M_{j}^{-1}$ is integrable $j=1, \ldots, l$. Proposition 4.6 holds more generally if $w\left|p_{k_{1}}(x)\right|^{-1 / k_{1}}$ is integrable. The borderline case arises when $w\left|p_{k_{1}}(x)\right|^{-1 / k_{1}}$ is barely nonintegrable. Typical for this is $p_{k}(x)=O\left(x^{k}\right)$ for $k$ in the lowest class. Thus if one is looking for absolutely continuous spectrum, it suffices to consider the lowest class alone, i.e. $\mathfrak{P}=\mathfrak{P}_{1}$. With some mild reindexing we are led to the characteristic Fourier polynomial in the even order case,

$$
\mathfrak{P}=\sum_{k=0}^{2 n}\left(c_{k}+h_{k}\right) x^{k} \lambda^{k}-z, \quad c_{2 n}=1=w .
$$

The corresponding operator $L$ on $\mathscr{L}^{2}([1, \infty))$ thus has the coefficients $p_{k}=$ $\left(c_{2 k}+h_{2 k}\right) x^{2 k}$ and $q_{j}=1 / 2\left(c_{2 j-1}+h_{2 j-1}\right) x^{2 j-1}$. For the perturbing terms we assume

$$
h_{k}, x h_{k}^{\prime}, x^{2} h_{k}^{\prime \prime}=o(1), \quad x^{-1} h_{k}, h_{k}^{\prime} \in \mathscr{L}^{2}, \quad x h_{k}^{\prime \prime} \in \mathscr{L}^{1} .
$$


In order to transform the system associated to $L$ into Levinson form we proceed as outlined in Section 2.3. The roots $\lambda_{i}$ of $\mathfrak{P}$ are clearly of the form

$$
\lambda_{i}=x^{-1}\left(\mu_{i}+\varphi_{i}\right)
$$

with $\left(\mu_{i}+\varphi_{i}\right)$ a root of $\sum\left(c_{k}+h_{k}\right) \mu^{k}$, and the dichotomy condition requires the roots of

$$
Q(\mu)=\sum c_{k} \mu^{k}
$$

to be distinct with distinct real parts. Here we have again absorbed the spectral parameter into $p_{0}$. By looking at the discriminant of $Q$ it is easy to see that for all but finitely many $z \in \mathbb{R}$ the polynomial $Q$ has distinct roots. This exceptional set will be ignored henceforth. Since the roots of $Q$ are distinct the correction terms $\varphi_{i}$ are analytic in $h_{1}, \ldots, h_{2 n}$. The matrix elements of $\left(T^{-1} T^{\prime}\right)_{i j}$, as they appear in the first diagonalization are explicitly computed in [3] and [8]. One finds

$$
\left(T^{-1} T^{\prime}\right)_{i j}=x^{-1}\left(t_{i j}+\tau_{i j}\right), \quad \tau_{i j}=o(1), t_{i j} \text { constant, } i \neq j,
$$

where $\tau_{i j}$ arises from the $h_{k}, x h_{k}^{\prime}, k=1, \ldots, 2 n$. The $t_{i j}$ depend only on the coefficients $c_{1}, \ldots, c_{2 k}$. Their precise value, however, is not important for us at the moment. If the constant matrix $D=\left(\operatorname{diag}\left(\mu_{i}\right)+t_{i j}\right)$ has distinct eigenvalues $\xi_{i}$, $i=1, \ldots, 2 n$, this also holds for the matrix $\widetilde{D}=\left(\operatorname{diag}\left(\mu_{i}+\varphi_{i}\right)+t_{i j}\right)$, at least for large $x$. Thus there exists an almost constant matrix $T_{3}$, which diagonalizes $\tilde{D}$. Then $-T^{-1} T^{\prime}$ is made up from terms $h_{k}^{\prime}$, because the $\varphi_{i}$ are analytic in the $h_{k}$. The transformed system has the form

$$
v^{\prime}=x^{-1}\left(\operatorname{diag}\left(\xi_{i}+\psi_{i}\right)+R\right) v
$$

The constant values $\xi_{i}$ are the eigenvalues of $D$ while the $\left(\xi_{i}+\psi_{i}\right)$ are the roots of $\tilde{D}$. Again the $\psi_{i}$ are analytic in the $h_{k}$, without constant terms. $R$ is made up from $-T_{3}^{-1} T_{3}^{\prime}$ as well as the $\tau_{i j}$. Thus $R$ is analytic in $x^{-1} h_{k}, h_{k}^{\prime}$. In particular we have $R_{i j} \in \mathscr{L}^{2}, R_{i i}=0$. A further diagonalization transforms this system into Levinson form. This diagonalization is achieved by a matrix of the form $(1+B)$ with $B_{i j}=\left(\lambda_{j}-\lambda_{i}\right)^{-1} R_{i j}$. Thus the contribution to the diagonal is integrable and thus negligible.

Since the factors $M_{j}=\left(\partial_{\lambda} \mathfrak{P}(\lambda)\right)\left(\lambda_{j}\right)$ satisfy $M_{j} \approx x$ the eigenfunctions of $L$ have the form

$$
y_{i}=x^{-\frac{1}{2}}\left(1+r_{1}(x)\right) \exp \int_{1}^{x}\left(\xi_{i}+\psi_{i}\right) d t, \quad r_{i}=o(1),
$$

provided the dichotomy condition can be shown. 
In order to determine the eigenvalues $\xi_{i}$ we use the fact that the differential equation $L y=z y$ is an Euler equation for $h_{k}=0$. With the Ansatz $x^{v}$ we get the indicial equation

$$
0=\sum c_{2 k} P_{k}(v)+i \sum c_{2 j-1} Q_{j}(v)=\tilde{Q}(v)
$$

where

$$
\begin{aligned}
P_{k}(v) x^{\nu} & =\left(x^{2 k}\left(x^{\nu}\right)^{(k)}\right. \\
& =v(v-1) \cdots(v-k+1)(v+k) \cdots(v+1) x^{v}
\end{aligned}
$$

and

$$
\begin{aligned}
Q_{j}(v) x^{v}= & \left.\frac{1}{2}\left(x^{2 j-1}\left(x^{\nu}\right)^{(j)}\right)^{(j-1)}+\left(x^{j}\left(x^{\nu}\right)^{(j-1)}\right)^{(j)}\right) x^{v} \\
= & \frac{1}{2} x^{\nu}[v(v-1) \ldots(v-j+1)(v+j-1) \cdots(v+1)+v(v-1) \\
& \cdots(v-j+2)(v+j) \cdots(v+1)] .
\end{aligned}
$$

As noted above, we may, excepting finitely many $z$ values, assume that this polynomial has distinct roots. Thus no additional factors appear and all eigenfunctions of $L y=$ $z y$ have the solutions $x^{v}$, with $v$ a root of the indicial equation. We are looking for solutions, which are square integrable and barely square integrable. For this reason write $v=\xi-\frac{1}{2}$. This also takes care of the factor $x^{-\frac{1}{2}}$ arising from $M_{1}^{-\frac{1}{2}}$. Now

$$
\begin{aligned}
& P_{k}\left(\xi-\frac{1}{2}\right)=\left(\xi-k+\frac{1}{2}\right)\left(\xi-k+\frac{3}{2}\right) \cdots\left(\xi+k-\frac{1}{2}\right), \\
& Q_{j}\left(\xi-\frac{1}{2}\right)=\left(\xi-j+\frac{3}{2}\right)\left(\xi-j+\frac{5}{2}\right) \cdots\left(\xi-j-\frac{3}{2}\right) .
\end{aligned}
$$

Note that the even (odd) order terms go with real (purely imaginary) coefficients, turning $\widetilde{Q}\left(i \xi-\frac{1}{2}\right)$ for $z \in \mathbb{R}$ into a polynomial with real coefficients. This of course has its parallel with the Fourier polynomial introduced above (2.15) and (2.16). If $\xi$ is a root of $Q(\xi)=\widetilde{Q}\left(\xi-\frac{1}{2}\right)=0$, the corresponding solution has the form

$$
x^{-\frac{1}{2}} x^{\xi}=c x^{-\frac{1}{2}} \exp \int_{1}^{x} \frac{\xi}{t} d t .
$$

This corresponds exactly to (2.24). Thus the eigenvalues of the matrix (diag $\mu_{i}+t_{i j}$ ) are just the $\xi_{i}$, the roots of $Q(\xi)=0$.

The corresponding Fourier polynomial $Q(i \xi)$ has for $z \in \mathbb{R}$ real coefficients. Again, excepting finitely many $z$ values it has distinct roots of the form $\alpha_{1} \pm$ $i \beta_{1}, \ldots, \alpha_{r} \pm i \beta_{r}, \gamma_{2 r+1}, \ldots, \gamma_{z n}$ with $\alpha_{i}, \beta_{i}, \gamma_{j}$ real. By analyticity and stability of the root pattern this presentation extends to the eigenvalues of $\tilde{D}$.

For spectral values $z+i \eta$ off the real axis the roots are approximately given by

$$
\lambda(z+i \eta)=\lambda(z)+i \eta\left(\partial_{\lambda} Q\right)^{-1}(\lambda(z)) .
$$


Thus the $z$-uniform dichotomy condition amounts to show that for any two roots $\xi_{1}, \xi_{2}$ of $\mathrm{Q}$ one has $\operatorname{Re} \xi_{1} \neq \operatorname{Re} \xi_{2}$. Actually, because of [5] it suffices to show this only for the purely imaginary roots $i \gamma_{2 r+1}(z), \ldots, i \gamma_{2 n}(z)$. For this it suffices that

$$
\partial_{\lambda} Q\left(\lambda_{i}\right) \neq \partial_{\lambda} Q\left(\lambda_{2}\right), \quad \lambda_{j}=i \gamma_{j}(z)
$$

for all $z$, which do not correspond to multiple roots. If these conditions were violated for $z$ with an accumulation point, analyticity would give $\partial_{\lambda} Q\left(\lambda_{2}(z)\right)$ or $Q\left(\lambda_{1}(z)\right)=$ $Q\left(\lambda_{2}(z)+C\right.$ for some constant $C$. By Bezout's theorem this is only possible for $\lambda_{1}=\lambda_{2}$. Thus the $z$-uniform dichotomy conditions hold outside a spectrally irrelevant set $\mathcal{E}$. This shows the following result.

Theorem 4.7. The minimal differential operator $T$ of order $2 n$ on $[1, \infty)$ with coefficients defined by (1.1) satisfying (4.20) and (4.21) is limit point, def $T=(n, n)$, at infinity. For any selfadjoint extension $H$ one has: $H$ has no singular continuous spectrum and $z$ belongs to the absolutely continuous spectrum of multiplicity $k$, if $Q$ has $2 k$ purely imaginary roots.

Proof. For the proof fix a spectral interval $I=\left[z_{0}-\varepsilon, z_{0}+\varepsilon\right]$ outside the exceptional set $\varepsilon$. Then for any $z+i \eta,|\eta|<\varepsilon$ suitably small, the $z+i \eta$ eigenfunction is given by

$$
y_{j}(x, z+i \eta)=x^{-\frac{1}{2}}\left(e_{j}+r_{j}(x)\right) \exp \left(\int_{1}^{x} t^{-1}\left(\xi_{j}(z+i \eta)+\psi_{j}(t)\right) d t\right) .
$$

If $\psi_{j}=i\left(\alpha_{j}+i \beta_{j}\right)$ with $\beta_{j}(z+i \eta)>0$ the corresponding eigenfunction is $z$-uniformly square integrable. For essentially imaginary eigenvalues $i \gamma_{j}$, however, we have

$$
i \gamma_{j}(z+i \eta)=i \gamma_{j}(z)-\eta\left(\partial_{\lambda} Q(\gamma)\right)^{-1}
$$

so that the eigenfunction is square integrable (not square integrable) if $\partial_{\lambda} Q(\gamma)>0$ $\left(\partial_{\lambda} Q(\gamma)<0\right)$. Since $Q$ is an even polynomial there are $r$ uniformly square integrable solutions and $(n-r)$ solutions, which are square integrable for $\eta>0$, but lose their square integrability as $\eta \rightarrow 0+$.

For odd order operators the analysis can proceed along the same lines. For $c_{2 n+1}=1$, however, one has def $T=(n+1, n)$.

4.3. Singular differential operators on bounded intervals. So far asymptotic integration has essentially been used to study the behavior of solutions on semi-infinite intervals. The following class of problems is devoted to differential operators with unbounded coefficients on bounded intervals. By using the decomposition method this can be reduced to the problem of one singularity. Thus we study the operator

$$
L y=w^{-1}\left(\sum_{i=0}^{n}\left(p_{k}(t) y^{(k)}(t)\right)^{(k)}\right), \quad p_{n}=1,
$$


on the interval $(0,1]$ and allow a regular singularity of the coefficients at 0 , i.e.

$$
\left|t^{2(n-k)} p_{k}(t)\right| \longrightarrow 0 \quad \text { as } t \rightarrow 0
$$

Otherwise we assume the coefficients to be differentiable. Replacing $p_{0}-z w$ by $p_{0}$ as usual with the systems vector $u=\left(y^{[0]}, y^{[1]}, \ldots, y^{[n]}, y^{[n+1]}, \ldots, y^{[2 n-1]}\right)$, we get

$$
u^{\prime}(t)=C(t) u
$$

where the non-zero matrix elements of $C$ are given by

$$
\begin{aligned}
C_{i, i+1} & =1, \quad i=1, \ldots, n, \\
C_{i, i+1} & =-1, \quad i=n+1, \ldots, 2 n, \\
C_{n+1, n-(i-1)} & =p_{n-i+1} .
\end{aligned}
$$

Transform this system to $[1, \infty)$ by $x=\frac{1}{t}$. Then

$$
\frac{d u}{d x}=\left(R-x^{-2} E\right) u(x),
$$

where the non-zero matrix elements of $R$ and $E$ are given by

$$
\begin{aligned}
R_{2 n-j, j+1} & =-x^{-2} p_{j}\left(x^{-1}\right), \quad 0 \leq j \leq n-1, \\
E_{j, j+1} & =1, \quad j=1, \ldots, n,
\end{aligned}
$$

and

$$
E_{j, j+1}=-1, \quad n+1 \leq j<2 n-1 .
$$

This system is almost a Jordan type system analyzed in [14], Section 1.10. Now let

$$
D=\operatorname{diag}\left(1, x^{-1}, \ldots, x^{-(2 n-1)}\right) \quad \text { and } \quad \varphi(x)=\exp \left(x^{-1} E\right) .
$$

Then

$$
\varphi^{\prime}(x)=x^{-2} E \varphi(x) \quad \text { and } \quad D^{-1} E D=x^{-1} E .
$$

The latter relation implies

$$
D^{-1} e^{E} D=\varphi
$$

The transformation $u=\varphi z$ easily gives

$$
z^{\prime}=\varphi^{-1} R \varphi z
$$

The further transformation $D z=w$ finally yields with (4.36), see Section 1.10.15 of [14],

$$
w^{\prime}=\left(D^{\prime} D^{-1}+e^{-E} D R D^{-1} e^{E}\right) w .
$$


This system is in Levinson form. The dichotomy condition is satisfied likewise, because

$$
D^{\prime} D^{-1}=-x^{-1} \operatorname{diag}((i-1)) .
$$

Thus, if

$$
\left(D R D^{-1}\right) \in \mathscr{L}^{1},
$$

the solution of (4.38) are essentially the integrals of

$$
w^{\prime}=-x^{-1}(i-1) w \quad \text { or } \quad w=c_{i} \cdot x^{-(i-1)}
$$

by Levinson's Theorem. More precisely one has

$$
u=\varphi D^{-1} w=D^{-1} e^{E} w .
$$

The matrix $\exp E$ is upper triangular with matrix elements

$$
\left(e^{E}\right)_{k, k+l}=\frac{1}{l !}(-1)^{k+l-1-n}
$$

if $k+l-1>n$ otherwise the value is just $(l !)^{-1}$. Thus the $k$-th solution is up to a sign just [14], Section 1.10.8,

$$
\begin{aligned}
u_{k}(x)= & \left(\frac{x^{-(k-1)}}{(k-1) !}+o\left(x^{-(k-1)}\right), \frac{x^{-(k-2)}}{(k-2) !}+o\left(x^{-(k-2)}\right), \ldots,\right. \\
& \left.1+o(1), o(x), o\left(x^{2}\right), \ldots, o\left(x^{2 n-k}\right)\right) .
\end{aligned}
$$

For our original solution this means

$$
\begin{gathered}
u_{k}(t)=\left(t^{k-1}+o\left(t^{k-1}\right),(k-1) t^{(k-2)}+o\left(t^{k-2}\right), \ldots,\right. \\
\left.1+o(1), o\left(t^{-1}\right), \ldots, o\left(t^{2 n-k}\right)\right) .
\end{gathered}
$$

Surprising about this is the fact that the higher quasiderivatives are of the order $o\left(t^{-l}\right)$. This means these terms are rather imprecisely known. This in turn has consequences in the implementation of the boundary conditions at 0 . The operator is limit circle at 0 , thus $T$ on $(0,1]$ has deficiency index $\operatorname{def} T=(2 n, 2 n)$ and the spectrum of any selfadjoint extension is discrete. In general the boundary conditions at 0 will have to be realized with the aid of solutions $T^{*} y=i y$ [19]. For Dirichlet boundary conditions, however, only $y_{2 n-1}, \ldots, y_{n}$ qualify.

Remark 4.8. The above approach can be generalized in many ways. Obviously the analysis extends to systems with first superdiagonals of the form $\left(t^{r_{1}}, t^{r_{2}}, \ldots, t^{r_{2 n-1}}\right)$, $r_{i} \geq 0$. Secondly further transformations may weaken the demands on $R$; see [14], Section 10 . 
Even though the above analysis is rather complete, we shall consider a particular example in order to understand the strange $o\left(t^{-l}\right)$ terms, which also appear with the solutions of an Euler equation.

Example $18\left((-1)^{n} y^{(2 n)}+c t^{-\alpha} y(t)=z y, \alpha<2 n\right)$. Following the above analysis we are led to the equation

$$
w^{\prime}=\left(-x^{-1} \operatorname{diag}(i-1)+S\right) w
$$

where

$$
\begin{aligned}
S_{i j} & =\left(e^{-E} D R D^{-1} e^{E}\right)_{i j} \\
& =\left(e^{-E}\right)_{i, 2 n}\left(\frac{1}{x^{2}} p_{0}\left(\frac{1}{x}\right)\right) e_{i j}^{E} x^{-(2 n-1)}, \\
& =C_{i j} c p_{o}\left(\frac{1}{x}\right) x^{-2 n-1},
\end{aligned}
$$

where $C_{j k}= \pm((2 n-i) !)^{-1}((j-1) !)^{-1}$ is a combinatorial factor. In order to determine the $k-t h$ solution $w$ of (12) consider the neutralized solution

$$
\tilde{w}=w \exp \mu_{k}(t)
$$

with

$$
\mu_{k}(x)=-\int_{1}^{x}(k-1) x^{-1}=-(k-1) \ln x
$$

and the associated integral equation for its $j$-th component $\tilde{w}_{j}$,

$$
\tilde{w}_{j}(x)= \begin{cases}\delta_{j k}+\int_{a}^{x}(s / x)^{j-k} \sum_{l} c_{j l} c s^{\alpha-(2 n+1)} \tilde{w}_{l}(s) d s, & j \geq k, \\ -\int_{x}^{\infty}(x / s)^{k-j} \sum_{l} c_{j l} c s^{-(2 n+1)+\alpha} \tilde{w}_{l}(s) d s, & j<k .\end{cases}
$$

This system will be solved by iteration with starting vector $\tilde{w}_{j}(x)=\delta_{j k}$. Then the first iteration gives

$$
\tilde{w}_{j}(x)= \begin{cases}\delta_{j k}+\left.c c_{j k}(\alpha-2 n+(j-k))^{-1} x^{k-j} t^{\alpha-2 n+(j-k)}\right|_{a} ^{x}, & j \geq k, \\ =c_{j k} c(\alpha-2 n+(j-k))^{-1} x^{-2 n+\alpha}, & j<k .\end{cases}
$$

These terms may also be taken as estimates of the o(1) terms in (4.42), because the order of $S_{j i}$ is independent of the indices and because Levinson's Theorem, respectively the iteration procedure, guarantees $\tilde{w}_{j}(x)=o(1)$ for $j \neq k$ with (1.3). 
Example 19. Consider an operator $L_{0}$ of the form $(1.1)$ on $(0, \infty)$ and let

$$
L=L_{0}+\sum_{l=1}^{n} c_{l}\left|x-x_{l}\right|^{-\alpha}, \quad \alpha<2 n, 0<x_{1}<x_{2}, \ldots<x_{k}
$$

Then the associated minimal operator $T$ has deficiency index

$$
\operatorname{def} T=(2 n k+n, 2 n k+n)
$$

and the absolutely continuous spectrum of $H$ is given by the absolutely continuous spectrum of $L_{0}$.

\section{References}

[1] H. Behncke, Finite dimensional perturbations. Proc. Amer. Math. Soc. 72 (1978), 82-84. MR 0503536 Zbl 0393.47017

[2] H. Behncke, Spectral analysis of fourth order differential operators II. Math. Nachr. 279 (2006), 73-85. MR 2193608 Zbl 1105.47041

[3] H. Behncke, Spectral theory of higher order differential operators. Proc. London Math. Soc. 92 (2006), 139-160. MR 2192387 Zbl 1099.34073

[4] H. Behncke, The remainder in asymptotic integration. Proc. Amer. Math. Soc. 136 (2008), 3231-3238. MR 2407088 Zbl 1169.34037

[5] H. Behncke, Asymptotically constant linear systems. Proc. Amer. Math. Soc. 138 (2010), 1387-1393. MR 2578530 Zbl 1196.34072

[6] H. Behncke, Spectral analysis of fourth order differential operators III. Math. Nachr. 283 (2010), 1558-1574. MR 2759794 Zbl 1200.47061

[7] H. Behncke and D. B. Hinton, Transformation Theory of Symmetric Differential Expressions. Adv. Differential Equations 11 (2006), 601-626. MR 2238021 Zbl 1160.34076

[8] H. Behncke and D. B. Hinton, Eigenfunctions, deficiency indices and spectra of odd order differential operators. Proc. London Math. Soc. (3) 97 (2008), 425-449. MR 2439668 Zbl 1158.34050

[9] H. Behncke and D. B. Hinton, Spectral theory of Hamiltonian systems with almost constant coefficients. J. Differential Equations 250 (2011), 1408-1426. MR 2737210 Zbl 1237.34141

[10] H. Behncke, D. Hinton, and C. Remling, The spectrum of differential operators of order $2 n$ with almost constant coefficients. J. Differential Equations 175 (2001), 130-162. MR 1849227 Zbl 0990.34070

[11] H. Behncke and F. O. Nyamwala, Spectral analysis of higher order differential operators with unbounded coefficients. Math. Nachr. 285 (2012), 56-73. MR 2864554 Zbl 06012475

[12] H. Behncke and C. Remling, Asymptotic integration of linear differential equations. J. Math. Anal. Appl. 210 (1997), 585-597. MR 1453193 Zbl 0883.47041 
[13] H. Behncke and C. Remling, Uniform asymptotic integration of a family of linear differential systems. Math. Nachr. 225 (2001), 5-17. MR 1827087 Zbl 0995.34045

[14] M. S. P. Eastham, The asymptotic solution of linear differential systems. Applications of the Levinson theorem. London Mathematical Society Monographs, New Series, 4. Clarendon Press, Oxford, 1989. MR 1006434 Zbl 0674.34045

[15] W. N. Everitt, Fourth-order Bessel-type special functions: a survey. In S. Elaydi, J. Cushing, R. Lasser, A. Ruffing, V. Papageorgiou, W. Van Assche (eds.), Difference equations, special functions and orthogonal polynomials. Proceedings of the international conference, Munich, Germany, July 25-30, 2005. World Scientific, Hackensack, NJ, 2007, 189-212. MR 2451169 Zbl 1221.33009

[16] W. N. Everitt and C. Markett, On a generalization of Bessel functions satisfying higher-order differential equations. J. Computational Appl. Math. 54 (1994), 325-349. MR 1321078 Zbl 0827.33003

[17] I. M. Glazman, Direct methods of qualitative spectral analysis of singular differential operators. Israel Programme for Scientific Translations, Jerusalem, and Oldbourne Press, London, 1965. MR 0190800 Zbl 0143.36505

[18] D. B. Hinton and J. K. Shaw, On the Titchmarsh-Weyl $M(\lambda)$-functions for linear Hamiltonian systems. J. Differential Equations 40 (1981), 316-342. MR 0621248 Zbl 0472.34014

[19] D. B. Hinton and A. Schneider, On the Titchmarsh-Weyl coefficients for singular S-Hermitian systems I. Math. Nachr. 163 (1993), 323-342. MR 1235076Zbl 0806.34023

[20] D. B. Hinton and A. Schneider, Titchmarsh-Weyl coefficients for odd order linear Hamiltonian systems. J. Spectral Mathematics 1 (2006), 1-36.

[21] M. A. Naimark, Linear differential operators II. Linear differential operators in Hilbert space. Ungar, New York, 1967. MR 0262880 Zbl 0227.34020

[22] C. Remling, Spectral analysis of higher order differential operators I. General properties of the M-function. J. London Math. Soc. (2) 58 (1998), 367-380. MR 1668199 Zbl 0922.34068

[23] C. Remling, Spectral analysis of higher order differential operators II. Fourth order equations. J. London Math. Soc. (2) 59 (1999), 188-206. MR 1688498 Zbl 0930.34061

[24] P. W. Walker, A vector-Matrix formulation for formally symmetric ordinary differential equations with applications to solutions of integrable square. J. London Math. Soc. (2) 9 (1974), 151-159. MR 0369792 Zbl 0308.34011

[25] J. Weidmann, Spectral theory of ordinary differential operators. Lecture Notes in Mathematics 1258. Springer Verlag, Berlin etc., 1987. MR 0923320 Zbl 0647.47052

Received February 24, 2012

Horst Behncke, Fachbereich Mathematik/Informatik, Universität Osnabrück, 49069 Osnabrück, Germany

E-mail: jones@mathematik.uni-osnabrueck.de

D.B. Hinton, Mathematics Department, University of Tennessee, Knoxville, TN 37996, U.S.A.

E-mail: hinton@math.utk.edu 